\title{
The Genetic Structure, Virulence, and Fungicide Sensitivity of Fusarium fujikuroi in Taiwan
}

\author{
Yu-Chia Chen, Ming-Hsin Lai, Chia-Yi Wu, Tsung-Chun Lin, An-Hsiu Cheng, Chin-Cheng Yang, Hsin-Yuh Wu, \\ Sheng-Chi Chu, Chien-Chih Kuo, Yea-Fang Wu, Guo-Cih Lin, Min-Nan Tseng, Yi-Chen Tsai, Chun-Chi Lin, \\ Chi-Yu Chen, Jenn-Wen Huang, Heng-An Lin, and Chia-Lin Chung
}

First, sixth, and eighteenth authors: Master Program for Plant Medicine, National Taiwan University, No. 1, Sec. 4, Roosevelt Rd., Taipei City 10617, Taiwan; second author: Crop Science Division, Taiwan Agricultural Research Institute, No. 189, Zhongzheng Rd., Wufeng District, Taichung City 41362, Taiwan; third, seventeenth, and eighteenth authors: Department of Plant Pathology and Microbiology, National Taiwan University, Taipei City; fourth author: Plant Pathology Division, Taiwan Agricultural Research Institute, Taichung City; fifth, tenth, and eleventh authors: Crop Environment Section, Tainan District Agricultural Research and Extension Station, No. 70, Muchang, Hsinhua District, Tainan City 71246, Taiwan; seventh author: Crop Environment Section, Taoyuan District Agricultural Research and Extension Station, No. 139, Sec. 2, Dongfu Rd., Xinwu District, Taoyuan City 32745, Taiwan; eighth author: Crop Environment Section, Miaoli District Agricultural Research and Extension Station, No. 261, Guannan Village, Gongguan Township, Miaoli County 36346, Taiwan; ninth author: Crop Environment Section, Taichung District Agricultural Research and Extension Station, No. 370, Songhuai Rd., Tatsuen Township, Changhua County 51544, Taiwan; twelfth author: Crop Environment Section, Kaohsiung District Agricultural Research and Extension Station, 2-6 Dehe Rd., Dehe Village, Changjhih Township, Pingtung County 90846, Taiwan; thirteenth author: Crop Environment Section, Hualien District Agricultural Research and Extension Station, No. 150, Sec. 2, Ji'an Rd., Ji'an Township, Hualien County 97365, Taiwan; fourteenth author: Crop Environment Section, Taitung District Agricultural Research and Extension Station, No. 675, Sec. 1, Zhonghua Rd., Taitung City 95055, Taiwan; and fifteenth and sixteenth authors: Department of Plant Pathology, National Chung Hsing University, No. 250, Kuokuang Rd., South District, Taichung City 40227, Taiwan.

Accepted for publication 19 January 2016.

\begin{abstract}
Chen, Y.-C., Lai, M.-H., Wu, C.-Y., Lin, T.-C., Cheng, A.-H., Yang, C.-C., Wu, H.-Y., Chu, S.-C., Kuo, C.-C., Wu, Y.-F., Lin, G.-C., Tseng, M.-N., Tsai, Y.-C., Lin, C.-C., Chen, C.-Y., Huang, J.-W., Lin, H.-A., and Chung, C.-L. 2016. The genetic structure, virulence, and fungicide sensitivity of Fusarium fujikuroi in Taiwan. Phytopathology 106:624-635.

The rice disease bakanae, caused by Fusarium fujikuroi Nirenberg, has been present in Taiwan for over a century. To better understand the genetic diversity and structure of $F$. fujikuroi, a set of 16 polymorphic simple sequence repeat (SSR) markers were newly developed and used to analyze $637 \mathrm{~F}$. fujikuroi isolates collected in 14 cities or counties around Taiwan from 1996 to 2013. On the basis of Bayesian clustering, the isolates were classified into four highly differentiated clusters: cluster B likely derived from the more widespread and genetically diversified clusters A or C, and cluster D was restricted to four cities or counties and

may have been introduced from unknown sources genetically distinct from clusters A, B, and C. The coexistence of both mating types (MAT1$1: M A T 1-2=1: 1.88)$ and the highly diversified vegetative compatibility groups (VCG) (16 VCG among the 21 assessed isolates) suggest the likelihood of sexual reproduction in the field. However, the biased mating type ratios and linkage disequilibrium in the population suggest nonrandom mating between individuals. A significant pattern of isolation by distance was also detected, which implies a geographical restricted gene flow and low dissemination ability of $F$. fujikuroi. Evaluation of 24 representative isolates on eight rice varieties revealed differential levels of virulence, however no clear pattern of specific variety $\mathrm{x}$ isolate interaction was observed. Investigations of the differences in virulence and fungicide sensitivity between 8 early isolates (1998 and 2002) and 52 recent isolates (2012) indicate the evolution of increased resistance to the fungicide prochloraz in F. fujikuroi in Taiwan.
\end{abstract}

Bakanae disease of rice, also known as foolish disease, is caused by the ascomycetous fungus Fusarium fujikuroi Nirenberg (teleomorph Gibberella fujikuroi (Sawada) Wollenw.). Bakanae disease was first described in 1828, and is now widespread in many ricegrowing areas (Mew and Gonzales 2002). Losses of 20 to 50, 15, and $3.7 \%$ due to the disease were reported in Japan, India, and Thailand, respectively (International Rice Research Institute Rice Knowledge Bank, http://www.knowledgebank.irri.org/). The infected plants are elongated and slender, with long, thin, chlorotic

Corresponding author: C.-L. Chung; E-mail address: clchung@ntu.edu.tw

H.-Y. Wu, S.-C. Chu, C.-C. Kuo, Y.-F. Wu, G.-C. Lin, M.-N. Tseng, Y.-C. Tsai, C.-C. Lin, C.-Y. Chen, J.-W. Huang, and H.-A. Lin contributed equally to this work.

*The $\boldsymbol{e}$-Xtra logo stands for "electronic extra" and indicates that three supplementary figures and five supplementary tables are published online.

http://dx.doi.org/10.1094/PHYTO-11-15-0285-R

(C) 2016 The American Phytopathological Society leaves, as well as distinctly wide leaf angles with stems. Severely infected seedlings die at early stages. In some cases, bakanae symptoms become invisible after the infected seedlings are transplanted into the field; the symptoms can appear again after the tillering stage. The infection eventually causes plant death or the development of poorly filled or empty grains (Chang 2003; Leslie and Summerell 2006).

The causal agent of bakanae disease was first named G. fujikuroi (Sawada) Wollenw. based on the morphology of its perithecia; its anamorph stage was previously named $F$. moniliforme (Ou 1985). Recent lines of evidence from morphological, physiological, and molecular phylogenetic analyses revealed that the fungi previously named $F$. moniliforme can be classified into different species. These Fusarium spp. now belong to the G. fujikuroi species complex (GFSC). Among the distinct mating populations (MP) (biological species) in the GFSC, MP-C (anamorph $F$. fujikuroi) has the ability to produce gibberellic acid $\left(\mathrm{GA}_{3}\right)$ and cause typical bakanae symptoms (Amatulli et al. 2010; Desjardins et al. 2000; Mohd Zainudin et al. 2008b; Voigt et al. 1995). In Taiwan, Hsu et al. (2013a) 
used local isolates of $F$. fujikuroi, F. proliferatum, and F. verticillioides for pathogenicity tests. In agreement with previous studies, the authors concluded that all $35 \mathrm{~F}$. fujikuroi isolates led to bakanae disease, whereas $F$. proliferatum and $F$. verticillioides only caused slight etiolation and stunting of seedlings.

F. fujikuroi can survive in infected rice seeds, tissues, and soil. Bakanae disease usually occurs when contaminated seeds are used for planting; therefore, the disease can be well managed by treating seeds with effective fungicides. However, the increasing occurrence and severity of bakanae disease have been reported in some areas of South and East Asia. In 1998, the disease caused serious losses in Pakistan (Khan et al. 2000). It has also caused increasing damage to Boro rice in Bangladesh since 2000 (Haq et al. 2011). During 2006 to 2014 , the disease was found with 1.2 to $40 \%$ incidence on different aromatic basmati rice cultivars in northern India (Gupta et al. 2014). In recent years, the incidence of bakanae disease in Chungnam province in South Korea reached up to 22 to $36.3 \%$, despite applications of chemicals for seed treatment (Kim et al. 2015). A disease outbreak was found in eastern Taiwan in 2009, with more than $10 \%$ incidence in the fields (Huang and Chu 2009). During 2010 to 2012, the disease became more prevalent all around Taiwan.

The development of effective disease management strategies largely relies on the knowledge of pathogen population biology. F. fujikuroi reproduces by sexual and asexual reproduction, and both ascospores and conidia play important roles in the disease cycle (Chang 2003; Ou 1985). As a heterothallic fungus, F. fujikuroi mating and meiotic recombinations occur only when its compatible mating partners coexist in a population (Snyder and Sun 1973; Watanabe and Umehara 1977). By using two primer pairs, GFmat1a/GFmat1b and GFmat2c/GFmat2d, targeting the conserved $\alpha$-domain and high-mobility-group domain, respectively, the MAT1-1 and MAT1-2 idiomorphs of F. fujikuroi were identified (Carter et al. 2008; Steenkamp et al. 2000). Hyphal fusion of vegetative hyphae allows fungi, particularly asexual ones, to increase genetic diversity. Vegetative compatibility group (VCG) analysis, used to analyze the ability of two cospecific isolates to form a stable and functional heterokaryon following hyphal anastomosis, has been widely used to investigate the phylogenetic lineages of F. fujikuroi (Carter et al. 2008; Mohd Zainudin et al. 2008a; Puhalla and Spieth 1985; Sunder and Satyavir 1998) and other Fusarium spp. (Elmer et al. 1999; Leslie 1993; Mirtalebi and Banihashemi 2014; Puhalla 1985). Molecular markers have also been used to determine the genetic diversity of $F$. fujikuroi. Amplified fragment length polymorphism (AFLP) analysis was used to investigate $172 \mathrm{~F}$. fujikuroi isolates collected from California in 2000 to 2003 (Carter et al. 2008), and universally primed polymerase chain reaction (UP-PCR) was used to examine $20 \mathrm{~F}$. fujikuroi isolates collected from Nueva Ecija and Laguna provinces in the Philippines during the 2006 wet season (Cumagun et al. 2011).

Although bakanae disease has been present in Taiwan for over a century (Tamura 2012), the overall genetic structure and diversity of F. fujikuroi in Taiwan have never been studied. The first aim of this study was to develop a species-specific PCR assay and a robust codominant marker system for identification and population genetic studies of $F$. fujikuroi. We developed microsatellite or simple sequence repeat (SSR) markers to detect variations at multiple loci because of the advantages of high reproducibility, high polymorphism, and codominance in inheritance (Abdel-Mawgood 2012). The newly developed markers were then used to examine the genotypes of early and recent $F$. fujikuroi isolates from different areas of Taiwan. Mating type and VCG analyses were also performed to understand the reproductive mode and the source of genetic variability. Using sets of representative isolates selected from the SSR analysis, we investigated the differences in virulence and fungicide sensitivity between early and recent $F$. fujikuroi populations.

\section{MATERIALS AND METHODS}

Collection, isolation, and culture of $\boldsymbol{F}$. fujikuroi. In total, 637 F. fujikuroi samples from the seeds, seedlings, and adult plants of 31 host rice varieties were used in the study (Supplementary Table $\mathrm{S} 1$ ). Most of the isolates were collected from the widely cultivated varieties, including Tainan 11 (TN11), Taitung 30 (TT30), Tainung 71 (TNG71), Taikeng 9 (TK9), and Kaohsiung 139 (KH139). The 62 isolates from 1996 to 2011 (the only old isolates we could obtain) were provided by the Taiwan Agricultural Research Institute, Tainan District Agricultural Research and Extension Station, and Taoyuan Agricultural Research and Extension Station. The 575 isolates from 2012 to 2013 were collected in a nationwide collaborative project conducted by all seven District Agricultural Research and Extension Stations in Taiwan. The seed samples, in a total of 298 batches, were mostly collected from major certified seed growers in different areas. The seedling and adult plant samples were plants with symptoms collected by pathologists when performing the bakanae disease survey in 131 nursery stations and randomly chosen paddy fields.

To isolate F. fujikuroi, rice seeds were directly placed on the FFC selective medium (10 grains per plate) and cultured at $28^{\circ} \mathrm{C}$ for several days. The infected stem tissues were excised into approximately $1-\mathrm{cm}$ segments, surface sterilized in $1 \% \mathrm{NaOCl}$ for $30 \mathrm{~s}$, rinsed with sterile distilled $\mathrm{H}_{2} \mathrm{O}\left(\mathrm{dH}_{2} \mathrm{O}\right)$ for $30 \mathrm{~s}$, and cultured on the FFC selective medium at $28^{\circ} \mathrm{C}$ for several days. The FFC medium was prepared from Komada's basal medium ( $20 \mathrm{~g}$ of galactose, $2 \mathrm{~g}$ of L-asparagine, $1 \mathrm{~g}$ of $\mathrm{K}_{2} \mathrm{HPO}_{4}, 0.5 \mathrm{~g}$ of $\mathrm{MgSO}_{4} \cdot 7 \mathrm{H}_{2} \mathrm{O}, 0.5 \mathrm{~g}$ of $\mathrm{KCl}$, $0.01 \mathrm{~g}$ of $\mathrm{Fe}-\mathrm{Na}$-EDTA, $1 \mathrm{~g}$ of $\mathrm{Na}_{2} \mathrm{~B}_{4} \mathrm{O}_{7} \cdot 10 \mathrm{H}_{2} \mathrm{O}$, and $20 \mathrm{~g}$ of agar in 1 liter $\mathrm{dH}_{2} \mathrm{O} ; \mathrm{pH} 4.5$ ) amended with cycloheximide at $1 \mathrm{ppm}$, dichloran at $2 \mathrm{ppm}$, iprodine at $1 \mathrm{ppm}$, thiabendazole at $5 \mathrm{ppm}$, flutolanil at $10 \mathrm{ppm}$, lithium chloride at $1,000 \mathrm{ppm}$, and chloramphenicol at 200 ppm after autoclaving (Hsu 2013). On FFC medium, most nontarget microorganisms are inhibited, and F. fujikuroi could be differentiated from other Fusarium spp. commonly associated with rice seeds. F. verticillioides and the morphologically indistinguishable $F$. proliferatum are inhibited; $F$. semitectum can grow but its fluffy colony is very different from the flat and spreading colony of $F$. fujikuroi (Hsu 2013). A single hyphal tip of F. fujikuroi was then transferred to a new half-strength potato dextrose agar (1/2 PDA) plate (Difco, BD Diagnostics) and cultured for further use. The isolates were preserved by the dry filter-paper technique (http:// blog.mycology.cornell.edu/2008/01/10/a-simple-way-to-preservefungal-cultures/). To avoid the loss or attenuation of virulence due to long-term storage, all early isolates (from 1996 to 2002) were inoculated on seeds of TNG71 and reisolated from 3-week-old infected seedlings.

The identity of the fungus was verified by morphological characteristics and analysis by PCR with a species-specific primer pair, FfTef-F (5'-ATCCTGACCAAGATCTGGCGGGGTATATCTCA-3') and FfTef-R (5'-GCTCAGCGGCTTCCTATTGTCGAATGTTTAG TTTG- $\left.{ }^{\prime}\right)$. The primers were designed on the basis of the translation elongation factor 1- $\alpha(T E F-1 \alpha)$ sequences of $F$. fujikuroi (GQ848522 and GQ848527), F. proliferatum (GQ848533 and GQ848537), F. incarnatum (JN092338), F. graminearum (GQ848544 and GQ848545), F. oxysporum (GQ848538 and GQ848539), and F. verticillioides (GQ848528 and GQ848532) retrieved from the nucleotide database of National Center for Biotechnology Information (Supplementary Fig. S1). Each PCR was performed in a $10-\mu \mathrm{l}$ reaction mixture containing $20 \mathrm{ng}$ of genomic DNA, $0.2 \mu \mathrm{M}$ FfTef-F, $0.2 \mu \mathrm{M}$ FfTef-R, and $5 \mu \mathrm{l}$ of $2 \times$ GoTaq Green Master Mix (Promega-M7122). The thermal cycling parameters were 1 cycle of $94^{\circ} \mathrm{C}$ for $5 \mathrm{~min}$; followed by 35 cycles of $94^{\circ} \mathrm{C}$ for $30 \mathrm{~s}, 72^{\circ} \mathrm{C}$ for $30 \mathrm{~s}$, and $74^{\circ} \mathrm{C}$ for $30 \mathrm{~s}$; and a final extension step of $74^{\circ} \mathrm{C}$ for $5 \mathrm{~min}$.

DNA extraction. For each $F$. fujikuroi isolate, the mycelium was taken from a 14-day-old culture actively grown on 1/2 PDA medium. The mycelium was homogenized by using $2010 \mathrm{Geno} / \mathrm{Grinder}$ (SPEX SamplePrep). Approximately $50 \mathrm{mg}$ of pulverized genomic DNA was extracted following a standard cetyltrimethylammonium 
bromide (CTAB) extraction protocol (Doyle and Doyle 1987), with $500 \mu \mathrm{l}$ of CTAB extraction buffer $(2 \%$ [wt/vol] hexadecyltrimethylammonium bromide, $1.4 \mathrm{M} \mathrm{NaCl}, 20 \mathrm{mM}$ EDTA [pH 8.0], $0.1 \mathrm{M}$ Tris- $\mathrm{HCl}$ [pH 8.0], $2 \%$ polyvinylpyrrolidone, and $0.2 \%$ [vol/vol] 2-mercaptoethanol), $400 \mu \mathrm{l}$ of chloroform/isoamyl alcohol (24:1, vol/vol), and $300 \mu \mathrm{l}$ of isopropanol. The chloroform extraction and ethanol precipitation of DNA were performed according to standard procedures.

SSR marker development and genotyping. SSR markers were developed on the basis of the draft genome sequences of F. fujikuroi B14 (Jeong et al. 2013). SSR motifs in the assembled contigs were identified by using Tandem Repeats Finder v.4.04 (Benson 1999). The primers were designed with Primer3 v0.4.0 (Untergasser et al. 2012), based on the conserved sequences flanking the SSR motifs that showed a repeating pattern of 2 to $4 \mathrm{bp}$, and the number of repeats ranged from 5 to 10 . Genotypic analyses were conducted following a modified fluorescence-based SSR genotyping method (Hayden et al. 2008). The PIGtailing (Brownstein et al. 1996), fluorescent dye labeling, PCR condition, and amplicon analysis were performed as described elsewhere (Chung et al. 2015). Amplicons labeled with different fluorescent dyes were multiplexed (VIC/FAM/NED/PET = 2:4:4:6), mixed with formamide and GeneScan-500 LIZ size standard (Applied Biosystems), and analyzed on the Applied BioSystems 3730xl DNA Analyzer at Genomics BioSci \& Tech. The sizes of amplicons were scored by using GeneMapper (v3.0; Applied Biosystems).

Mating type analysis. The mating type of each F. fujikuroi isolate was determined by modified multiplex PCR (Steenkamp et al. 2000). Each PCR was performed in a 10- $\mu$ l reaction mixture containing $20 \mathrm{ng}$ of genomic DNA, $1 \mu \mathrm{M}$ GFmat $1 \mathrm{a}, 1 \mu \mathrm{M}$ GFmat $1 \mathrm{~b}$, $1 \mu \mathrm{M}$ GFmat2c, $1 \mu \mathrm{M}$ GFmat2d, and $5 \mu \mathrm{l}$ of Taq DNA polymerase $2 \times$ Master Mix Red (Ampliqon). The thermal cycling parameters were 1 cycle of $94^{\circ} \mathrm{C}$ for $5 \mathrm{~min}$; followed by 35 cycles of $92^{\circ} \mathrm{C}$ for $30 \mathrm{~s}$, $55^{\circ} \mathrm{C}$ for $30 \mathrm{~s}$, and $72^{\circ} \mathrm{C}$ for $45 \mathrm{~s}$; and a final extension step of $72^{\circ} \mathrm{C}$ for $5 \mathrm{~min}$. The PCR products were loaded in $1.5 \%$ agarose gels in $1 \times$ Tris-borate-EDTA buffer and run for $25 \mathrm{~min}$ at $100 \mathrm{~V}$. Gels were stained with ethidium bromide $\left(5 \times 10^{-4} \mathrm{mg} / \mathrm{ml}\right)$ for $10 \mathrm{~min}$, destained in double-distilled $\mathrm{H}_{2} \mathrm{O}\left(\mathrm{ddH}_{2} \mathrm{O}\right)$ for $10 \mathrm{~min}$, then photographed by using Gel Documentation-Videgel VGIS-4 (Top Bio Co.).

VCG analysis. To understand the VCG diversity of $F$. fujikuroi, 21 isolates were assigned to VCG based on the generation and complementation tests of nitrate-nonutilizing (nit) mutants, as described (Correll et al. 1987). The 21 isolates were a subset of the 60 representative $F$. fujikuroi isolates used in virulence test II (described in the "Virulence test" section below). They were chosen to represent both MAT1-1 and MAT1-2 isolates from the four SSR genetic groups in different regions of Taiwan (Supplementary Table S2: northwest $=$ Taoyuan, central west $=$ Miaoli and Taichung, southwest $=$ Tainan, central east $=$ Hualien, and southeast $=$ Taitung). F. fujikuroi isolates were cultured on minimal medium (MM) $\left(1 \mathrm{~g}\right.$ of $\mathrm{KH}_{2} \mathrm{PO}_{4}, 0.5 \mathrm{~g}$ of $\mathrm{Mg}_{4} \cdot 7 \mathrm{H}_{2} \mathrm{O}, 0.5 \mathrm{~g}$ of KCl, $2 \mathrm{~g}$ of $\mathrm{NaNO}_{3}$, sucrose at $5 \mathrm{~g} /$ liter, and $20 \mathrm{~g}$ of agar in 1 liter of $\mathrm{dH}_{2} \mathrm{O}$ ) or PDA amended with potassium chlorate at $15 \mathrm{~g} /$ liter. Colonies with abundant aerial mycelium were considered nit mutants. The nit mutants were classified into nit 1 , nit 3 , and NitM classes by subculturing on each of the four phenotyping media containing different nitrogen sources: (i) MM plus sodium nitrate at $2 \mathrm{~g} /$ liter, (ii) MM plus sodium nitrite at $0.5 \mathrm{~g} / \mathrm{liter}$, (iii) $\mathrm{MM}$ plus hypoxanthine at $0.2 \mathrm{~g} /$ liter, and (iv) MM plus ammonium tartrate at $1 \mathrm{~g} /$ liter. Pairwise complementation tests were conducted separately for a set of 13 representative isolates from all around Taiwan and a set of 8 isolates from Taichung. According to the results, pairs of isolates from different VCG were then tested for their compatibility. For each pairwise combination, complementary nit mutants of the two isolates (nit 1 by NitM or nit3 by NitM) were inoculated on MM plus sodium nitrate at $2 \mathrm{~g} /$ liter, approximately $1.5 \mathrm{~cm}$ apart from each other. Plates were incubated at $25^{\circ} \mathrm{C}$ under a cycle of 12 and $12 \mathrm{~h}$ of normal light and darkness, respectively. Abundant aerial mycelium growing out from the interface indicated heterokaryon formation between two isolates belonging to the same VCG. Complementary nit mutants from the same isolate were also paired to verify the heterokaryon self-compatibility. Pairings of all the combinations were repeated three times.

Virulence test. Two separate experiments, virulence tests I and II, were performed to determine the virulence of representative F. fujikuroi isolates and the resistance or susceptibility of selected rice varieties. The representative isolates were chosen to include those in the four SSR genetic groups and from different geographical areas. Virulence test I consisted of 24 representative isolates ( 1 to 3 isolates per city or county) from 2012 inoculated on eight rice varieties, including two indica varieties (Budda and IR7858112-3-2-2) and six japonica varieties (Toyonishiki, Koshihikari, KH139, TNG71, Taikeng 16 [TK16], and TN11). Two independent trials were conducted from 28 July to 18 August and 7 to 28 November 2013. The eight varieties were used to represent different levels of susceptibility to bakanae disease on the basis of their performance in two preliminary trials, in which 141 and 729 rice accessions from the germplasm were inoculated with $F$. fujikuroi Ff266 at $10^{3}$ spores/ml (M.-H. Lai, unpublished data). In virulence test II, 8 early isolates (from Taichung in 1998 and 2000) and 52 recent representative isolates (from 2012) were inoculated on three principal rice varieties at different times (Tainung 67 [TNG67], TN11, and TNG71). The set of 52 recent representative isolates consisted of 1 to 3 isolates per cluster from each city or county; the only exception was Nantou, from which only one isolate from cluster B was included. Two independent trials were conducted from 16 June to 7 July 2014. In Taiwan, the principal rice varieties were TNG67 during 1979 to 1998 and TN11 from 2005 until now, accounting for up to 75 and $50 \%$ of the total rice cultivation area, respectively. During 2003 to 2010, TNG71 was the most widely cultivated aromatic rice in central Taiwan (including Taichung) (aromatic varieties account for approximately $3 \%$ of the total rice cultivation area, and TNG71 accounts for 1 to $2 \%$ ). (Rice Registered Varieties Database, http:// tris.tari.gov.tw:8080/index.htm).

The inoculation method was conducted as previously described (Hsu et al. 2013b). F. fujikuroi was cultured on $1 / 2$ PDA at $25^{\circ} \mathrm{C}$ under a cycle of $12 \mathrm{~h}$ and $12 \mathrm{~h}$ of normal light and darkness, respectively. The spores were dislodged in sterile $\mathrm{dH}_{2} \mathrm{O}$, filtered through Kimwipes paper, and adjusted to a concentration of $10^{5}$ spores $/ \mathrm{ml}$. Before inoculation, rice seeds were surface disinfected in distilled water at $60^{\circ} \mathrm{C}$ for $10 \mathrm{~min}$, then immersed in running water for 3 days. The pregerminated seeds were soaked in the spore suspension or sterile $\mathrm{dH}_{2} \mathrm{O}$ and shaken for $1 \mathrm{~h}$. Seeds treated with sterile $\mathrm{dH}_{2} \mathrm{O}$ were used as healthy controls. The treated seeds were grown in Akadama soil and cultivated in a walk-in incubator at $28^{\circ} \mathrm{C}$ and $90 \%$ relative humidity under a 12 -h photoperiod. In each independent inoculation trial, we had 18 to 30 seedlings for each treatment (three pots per treatment, 6 to 10 seedlings/pot). Disease severity was evaluated on day 21 postinoculation. Each infected seedling was compared with healthy ones and rated on a modified 0 -to-3 scale (Hsu et al. 2013b), where $0=$ symptomless; $1=$ seedling with one type of the following symptoms: stem or internode elongation, large leaf angle, or seedling slender and pale; $2=$ seedling with complex symptoms; and 3 = seedling death. The overall disease severity index (DSI) was calculated as DSI $=[($ scale $\times$ number of seedlings at the scale $) /($ maximum scale $\times$ total number of seedlings $)] \times$ $100 \%$. To assess levels of virulence for each $F$. fujikuroi isolate on different rice varieties, data (DSI) from different rice varieties were analyzed by using JMP Pro 10 (SAS Institute Inc.) by fitting a linear model with "rice varieties" and "replications" as variables. The coefficients of the variables were estimated by the least squares method, and the least squares means were tested by Tukey-Kramer honestly significant difference (HSD) test at $P<0.05$. The 60 representative isolates in virulence test II belonged to 35 haplotypes. To assess the levels of virulence of the 35 haplotypes, data were analyzed as described above, with "haplotypes" and "replications" as variables. 
Fungicide sensitivity analysis. Inhibition effects of prochloraz and tebuconazole on spore germination and mycelial growth of 60 representative $F$. fujikuroi isolates (the same as the set of isolates used in virulence test II) were tested. For spore germination tests, each isolate was previously cultured on $1 / 2 \mathrm{PDA}$, and the spores were dislodged in $1 / 2$ potato dextrose broth (PDB), filtered, then adjusted to the concentration of $10^{5}$ spores $/ \mathrm{ml}$ in $1 / 2 \mathrm{PDB}$. The spore suspension $(10 \mu \mathrm{l})$ was mixed with an equal volume of $\mathrm{ddH}_{2} \mathrm{O}$ or a fungicide (final concentrations of prochloraz $=0,1,10$, and $100 \mathrm{ppm}$ and final concentrations of tebuconazole $=0,0.1,1$, and $10 \mathrm{ppm}$ ). The mixture was placed on a glass depression slide and incubated in a moist chamber for $12 \mathrm{~h}$ under normal light. For each treatment, the germination rate was determined by microscopic examination of 200 spores. A germinated spore was defined as the spore with a protruding germ tube twofold longer than its largest diameter. Two independent germination tests were performed.

For mycelial growth tests, several drops of a 4- $\mu$ l spore suspension $\left(10^{5}\right.$ spores $/ \mathrm{ml}$ in $\left.\mathrm{ddH}_{2} \mathrm{O}\right)$ were inoculated on $1 / 2 \mathrm{PDA}$. After $24 \mathrm{~h}$ of incubation at $25^{\circ} \mathrm{C}$ under a cycle of $12 \mathrm{~h}$ and $12 \mathrm{~h}$ of normal light and darkness, respectively, agar discs were cut out with a sterilized cork borer (4 $\mathrm{mm}$ in diameter) from the inoculated spots and then transferred to the $1 / 2 \mathrm{PDA}$ plates amended with $\mathrm{ddH}_{2} \mathrm{O}$ or a fungicide (final concentrations of prochloraz $=0,1,10$, and $100 \mathrm{ppm}$ and final concentrations of tebuconazole $=0,0.1,1$, and $10 \mathrm{ppm}$ ). Colony diameters were measured after 7 days of incubation at $25^{\circ} \mathrm{C}$ in darkness. The experiment was conducted in two independent trials, with four plates per treatment per trial.

Percentage of inhibition in germination rate or colony size was calculated as percent inhibition $=\left[\left(\right.\right.$ response with $\mathrm{ddH}_{2} \mathrm{O}-$ response with fungicide $) /\left(\right.$ response with $\left.\left.\mathrm{ddH}_{2} \mathrm{O}\right)\right] \times 100 \%$. The $50 \%$ effective dose $\left(\mathrm{EC}_{50}\right)$ value was estimated by regressing the inhibition response data against the logarithm-transformed concentrations of the fungicide. The average $\mathrm{EC}_{50}$ values for different genetic clusters (as mentioned in the Results) were analyzed by using JMP Pro 10 by fitting a linear model with "genetic clusters" and "replications" as variables. The $\mathrm{EC}_{50}$ values for early and recent isolates were analyzed by using "collection years" and "replications" as variables. The coefficients of the variables were estimated by the least squares method, and the least squares means were tested by the TukeyKramer HSD test at $P<0.05$. The average $\mathrm{EC}_{50}$ values for the 35 haplotypes were analyzed as described above, with "haplotypes" and "replications" as variables.

Microsatellite data analysis. In total, 637 isolates were genotyped for the 16 newly developed SSR markers. For each of the 16 SSR loci, number of alleles, major allele frequency, number of genotypes, genetic diversity, and polymorphism information content (PIC) were analyzed by using PowerMarker v3.25 (Liu and Muse 2005). Linkage disequilibrium between pairs of markers was tested with FSTAT v2.9.3.2 (Goudet 2001). The significance level was adjusted by Bonferroni correction for multiple comparisons.

The most probable number of genetic clusters $(K)$ in the $F$. fujikuro $i$ population was determined by Bayesian genetic clustering analyses in STRUCTURE 2.3.4 (Pritchard et al. 2000), under the assumptions of admixture and correlated or uncorrelated allele frequencies. Each run was done for $K=1$ to 10 after 500,000 burn-in iterations and 500,000 Markov chain Monte Carlo estimation iterations. Each run was carried out 20 times to ensure consistency of the results. An appropriate number of $K$ was selected based on the $\Delta K$ method by Evanno et al. (2005) and the reproducibility of clustering patterns. The assignment of isolates to hypothetical genetic clusters in different replicated runs was carefully checked. The optimal number of $K$ was also assessed using the function find.clusters with the adegenet package (Jombart 2008) in R (R Development Core Team 2011). It runs successive $K$-means clustering with increasing values of $K$ and computes the Bayesian Information Criterion (BIC) of the model. Discriminant analysis of principal components (DAPC) was also implemented using the function dapc in adegenet in $\mathrm{R}$. The optimal $K$ inferred from the STRUCTURE analysis was used as the prior information for isolate grouping. Mean number of alleles per locus $\left(N_{\mathrm{A}}\right)$ and allelic richness averaged over loci $\left(A_{\mathrm{R}}\right)$ (rarefactionbased estimation) were calculated with use of FSTAT v2.9.3.2 (Goudet 2001).

To assess the genetic diversity and clonality, GenAlEx 6.5 (Peakall and Smouse 2006) was used to identify distinct multilocus haplotypes in the entire Taiwan population, the presumed clusters, and the 14 regional subpopulations in different cities or counties. To reduce sampling bias, subsequent analyses were conducted with the clone-corrected dataset. The clone-corrected dataset consisted of only one isolate per clone (a clone was defined as the samples with identical haplotype, mating type, and VCG and originated from the same host rice variety at the same isolation stage from the same collection site and year). The genotypic diversity and the number or frequency of unique haplotypes within a population were computed by use of Multilocus v1.3 (Agapow and Burt 2001). To determine the degree of genetic differentiation, a modified Wright's $F_{\mathrm{ST}}$ for haploids called $\theta$ was used. Pairwise estimates of $\theta$ between clusters were calculated by in Multilocus v1.3 with 1,000 randomizations (Agapow and Burt 2001). Analysis of isolation by distance (IBD) between pairs of individuals (Rousset 2000) involved a Mantel's test, with 1,000 permutations in the ISOLDE program implemented in Genepop 4.2 (Raymond and Rousset 1995).

The difference between MAT1-1 and MAT1-2 frequencies was investigated by the $\chi^{2}$ goodness of fit test in R-Studio (R Development Core Team 2011). The effective population number $\left(N_{e(m t)}\right)$ was calculated by the equation $N_{e(m t)}=\left(4 N_{\text {MAT1-1 }} N_{\text {MAT1-2 }}\right) /\left(N_{M A T 1-1}+\right.$ $N_{M A T 1-2}$ ), where $N_{M A T 1-1}$ was the number of $F$. fujikuroi isolates carrying MAT1-1 and $N_{M A T 1-2}$ was the number of isolates carrying MAT1-2 (Leslie and Klein 1996). To assess whether a population was in linkage equilibrium, the clone-corrected haplotype data were used to perform multilocus linkage equilibrium tests (Brown et al. 1980) in Multilocus v1.3 with 1,000 randomizations. The index of association $\left(I_{A}\right)$ and the modified measure of multilocus linkage equilibrium $\left(\bar{r}_{d}\right)$ (standardized for the number of loci) were generated (Agapow and Burt 2001).

\section{RESULTS}

Marker development. The draft genome of $F$. fujikuroi comprised 455 contigs (Jeong et al. 2013). We initially designed 48 markers targeting the SSR regions in 48 randomly chosen contigs. The markers were analyzed for six $F$. fujikuroi isolates collected from six different cities or counties of Taiwan: isolates Ff259, Ff266, Ff267, Ff615, Ff677, and Ff783. A set of 16 SSR markers was selected for subsequent analyses (Supplementary Table S3), on the basis of polymorphism, PCR amplification efficiency, and discriminating capacity ( $>2$ bp difference between alleles). We also sequenced the amplicons of one dominant allele and one rare allele for each marker by the dideoxy termination method, and confirmed that all of these markers were locus specific and the differences in amplicon lengths conformed to the expected allelic variations. Analysis of $637 \mathrm{~F}$. fujikuroi isolates sampled across Taiwan at the 16 SSR markers revealed the presence of 140 alleles, ranging from 4 alleles at the SSR46 and SSR59 loci to 18 alleles at the SSR57 locus (Table 1; Supplementary Table S4). One allele alone per SSR locus was detected for all of the isolates, indicating that all samples were haploid. No significant linkage disequilibrium was observed between pairs of markers. There were 11 markers with PIC ranging from 0.18 to 0.545 and 5 markers with PIC ranging from 0.05 to 0.08 (SSR19, SSR33, SSR41, SSR42, and SSR47). Most rare alleles were found in cluster D (see below). To avoid potential effects from loci with low PIC values, we performed genetic analyses using the datasets with and without the five lowPIC markers, and inclusion of these markers did not seem to affect the clustering results or the among-cluster differences in genetic diversity and differentiation. Because inclusion of the five low-PIC markers resulted in higher resolution of clustering patterns, we decided to keep them for subsequent analyses in the study. 
Population structure and diversity. The results of Bayesian clustering analysis, BIC, and DAPC are shown in Figure 1. Similar results were obtained assuming correlated or uncorrelated allele frequencies. A peak at $K=2$ was observed in the $\Delta K$ plot (Fig. 1A) whereas, in the STRUCTURE bar plot (Fig. 1B), discernible genetic clusters were observed at $K=2,3$, and 4 . With STRUCTURE constrained to estimate 2,3 , or 4 clusters, the clustering pattern could only be consistently replicated for $K=4$ (same isolates clustered together in different runs), which suggests that $K=4$ is the most supported clustering value. The optimal clustering solution could not be identified by BIC, because the lowest BIC value was not found (Fig. 1C).

The four presumed genetic clusters (i.e., A, B, C, and D) were well separated and highly differentiated, based on DAPC (Fig. 1D)

TABLE 1. Summary statistics of the 16 simple sequence repeat (SSR) markers used to assess the 637 isolates of Fusarium fujikuroi in Taiwan

\begin{tabular}{|c|c|c|c|c|c|c|}
\hline SSR locus & $N^{\mathrm{x}}$ & Allele range $(b p)^{y}$ & Major allele amplicon (bp) & Major allele frequency & Gene diversity & $\mathrm{PIC}^{\mathrm{z}}$ \\
\hline 03 & 5 & $495-508$ & 495 & 0.83 & 0.29 & 0.27 \\
\hline 17 & 16 & $502-591$ & 542 & 0.62 & 0.57 & 0.53 \\
\hline 19 & 11 & $472-570$ & 478 & 0.97 & 0.07 & 0.07 \\
\hline 33 & 7 & $228-245$ & 239 & 0.97 & 0.05 & 0.05 \\
\hline 41 & 6 & $258-286$ & 258 & 0.97 & 0.06 & 0.06 \\
\hline 42 & 6 & $420-430$ & 428 & 0.96 & 0.09 & 0.08 \\
\hline 46 & 4 & $445-451$ & 447 & 0.84 & 0.26 & 0.23 \\
\hline 47 & 10 & $269-298$ & 273 & 0.96 & 0.08 & 0.08 \\
\hline 52 & 12 & $461-500$ & 471 & 0.77 & 0.37 & 0.34 \\
\hline 54 & 6 & $460-506$ & 460 & 0.82 & 0.30 & 0.27 \\
\hline 56 & 9 & $432-461$ & 439 & 0.68 & 0.48 & 0.43 \\
\hline 57 & 18 & $211-285$ & 240 & 0.57 & 0.59 & 0.54 \\
\hline 59 & 4 & $208-224$ & 222 & 0.62 & 0.49 & 0.38 \\
\hline 62 & 13 & $397-437$ & 402 & 0.80 & 0.33 & 0.30 \\
\hline 64 & 7 & $445-458$ & 451 & 0.90 & 0.19 & 0.18 \\
\hline 66 & 6 & $433-453$ & 441 & 0.66 & 0.47 & 0.38 \\
\hline
\end{tabular}

$x$ Number of alleles.

y Size of polymerase chain reaction product.

${ }^{\mathrm{z}}$ Polymorphism information content.
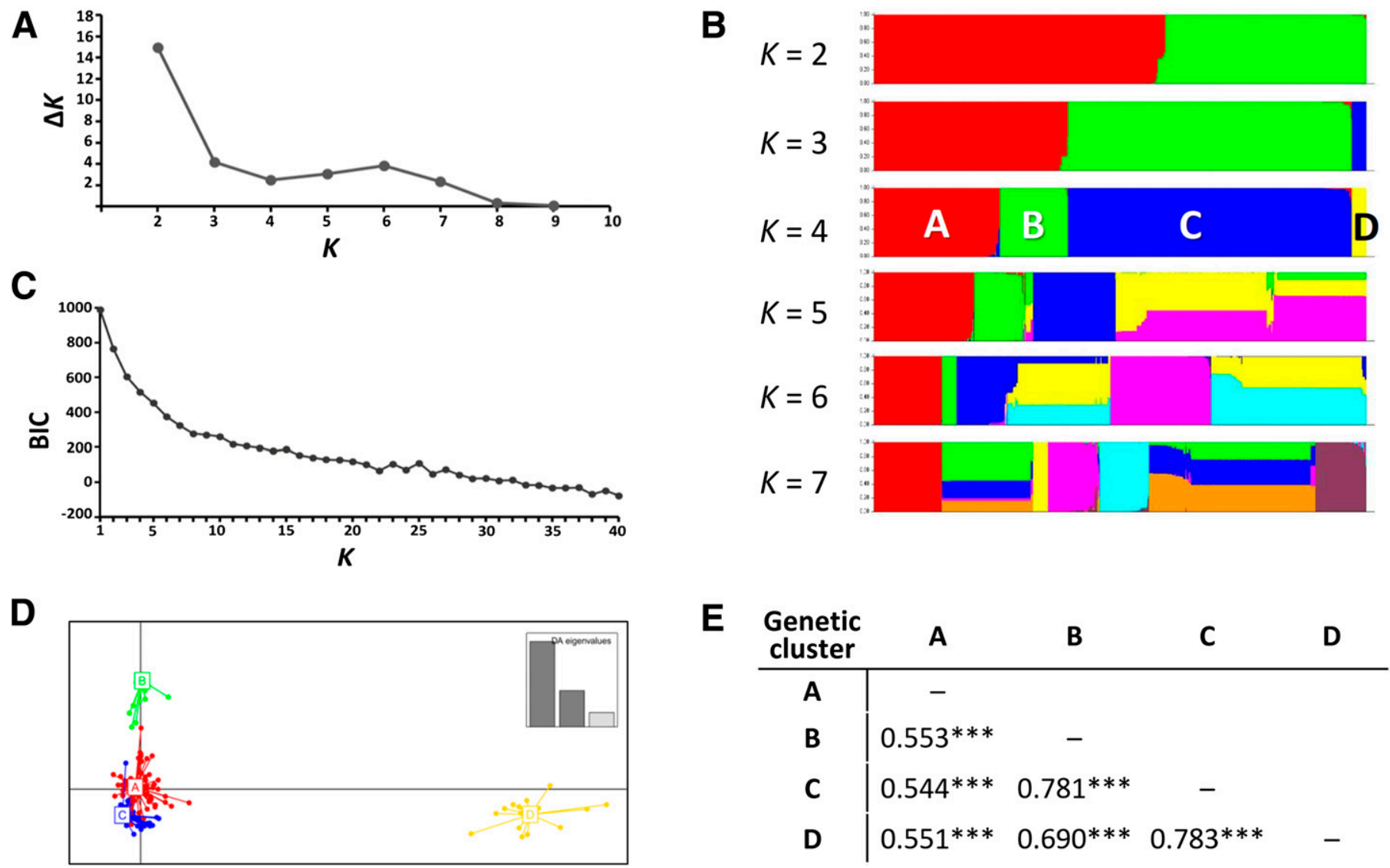

Fig. 1. Estimated Fusarium fujikuroi population structure in Taiwan by Bayesian genetic clustering analysis. $K$ is the number of presumed genetic clusters. A, The $\Delta K$ plot. B, STRUCTURE bar plot at $K=2$ to 7 . The clustering pattern could only be consistently replicated for $K=4$. Each bar represents an individual $F$. fujikuroi isolate. The colors represent different genetic clusters, and the lengths of the colored segments in a bar represent the estimated membership proportions of that individual to each cluster. C, Bayesian Information Criterion (BIC) plot. D, Discriminant analysis of principal components (DAPC) for the presumed four clusters inferred by STRUCTURE analysis. The scatterplot shows only the first two principal components, accounting for $80 \%$ of the total variance. DAPC eigenvalues are illustrated in the enclosed bar plot. E, Pairwise $\theta$ values between the four clusters inferred by STRUCTURE analysis. 
and pairwise $\theta$ values ( 0.544 to $0.784, P<0.001)$ (Fig. 1E). Cluster $\mathrm{D}$ was the most distantly related to the other clusters (Fig. 1D). The differentiation between cluster A and clusters $B, C$, and $D(\theta=0.544$ to 0.553 ) was not as high as that between clusters $\mathrm{B}, \mathrm{C}$, and D $(\theta=0.690$ to 0.784$)$. Multilocus analysis revealed 154 unique haplotypes, including 73, 21, 43, and 17 haplotypes in clusters A, B, $\mathrm{C}$, and D, respectively (Table 2). No shared haplotype was found between clusters. The computed values of the number or frequency of unique haplotypes, genotypic diversity, $N_{\mathrm{A}}$, and $A_{\mathrm{R}}$ are presented in Table 2. Cluster D had a high level of genetic diversity (genotypic diversity $=0.98, N_{\mathrm{A}}=4.94, A_{\mathrm{R}}=3.90$ ), despite its small sample size. Clusters A, B, and $\mathrm{C}$ had different levels of genetic diversity: cluster A (genotypic diversity $\left.=0.94, N_{\mathrm{A}}=5.63, A_{\mathrm{R}}=2.11\right)>>$ cluster $\mathrm{B}$ (genotypic diversity $\left.=0.80, N_{\mathrm{A}}=1.69, A_{\mathrm{R}}=1.38\right)>$ cluster $\mathrm{C}$ (genotypic diversity $=0.72, N_{\mathrm{A}}=3.00, A_{\mathrm{R}}=1.37$ ). Analysis of IBD revealed a significant relationship between spatial distance and genetic similarity for the entire Taiwan population and the four genetic clusters. Slopes of the regression lines were $0.083(P=0)$ for all Taiwan, $0.04(P=0.153)$ for cluster A, $0.145(P=0.047)$ for cluster $\mathrm{B}, 0.063(P=0.103)$ for cluster $\mathrm{C}$, and $0.083(P=0.157)$ for cluster D.

The composition of the 154 haplotypes and their geographical distributions are shown in Supplementary Table S5. In total, 140 haplotypes each contained 1 to 5 isolates, and 10 haplotypes each contained 6 to 16 isolates. Four predominant haplotypes were observed: H065 contained 155 isolates from 13 cities or counties,
H064 contained 115 isolates from 14 counties or cities, H145 contained 37 isolates from 8 cities or counties, and H039 contained 34 isolates from 7 cities or counties.

Geographical and temporal distribution of genetic clusters. The distributions of different genetic clusters were plotted on a map of Taiwan (Fig. 2). Clusters A, B, and C were widely distributed in Taiwan (clusters A and B from 13 counties and cluster C from 14 counties), whereas cluster D was sampled from only 4 counties, including Taoyuan, Miaoli, Hualien, and Taitung. Isolates of different clusters could be detected in all counties but Chiayi. The 15 isolates from Chiayi all belonged to cluster $\mathrm{C}$.

The composition of different genetic clusters in the F. fujikuroi population over time is shown in Table 2 . Because most of the early isolates available were from Taichung, temporal changes in population composition were examined for the overall Taiwan population and for the local population in Taichung. In the whole population, clusters A and C contained isolates from all time periods (1996 to 2002, 2009 to 2011, and 2012 to 2013) but clusters B and D contained isolates from only recent years (after 2009). The same pattern was observed in Taichung, except that cluster D was absent in this area.

Mating type and linkage equilibrium. The observed ratio of MAT1-1/MAT1-2 isolates was 221/416 (1:1.88) in Taiwan. Both mating types were commonly found in the four genetic clusters and in different areas across Taiwan (Table 3; Fig. 2). MAT1-1 and MAT1-2 were identified in most cities or counties; however, the isolates from Chiayi and Nantou were all MAT1-2. From the $\chi^{2}$ test,

TABLE 2. Genetic diversity (GD) and temporal distribution of the four genetic clusters of Fusarium fujikuroi in Taiwan

\begin{tabular}{|c|c|c|c|c|c|c|c|c|c|c|c|c|c|}
\hline \multirow[b]{2}{*}{ Clusterv $^{v}$} & \multirow[b]{2}{*}{$N^{\mathrm{w}}$} & \multicolumn{2}{|c|}{$\begin{array}{c}\text { Unique } \\
\text { haplotypes }^{\mathrm{t}}\end{array}$} & \multirow[b]{2}{*}{ GD } & \multirow[b]{2}{*}{ GD $(\text { corr })^{\mathrm{x}}$} & \multirow[b]{2}{*}{$N_{\mathrm{A}^{\mathrm{y}}}$} & \multirow[b]{2}{*}{$A_{\mathrm{R}}{ }^{\mathrm{z}}$} & \multicolumn{3}{|c|}{ Overall Taiwan ${ }^{u}$} & \multicolumn{3}{|c|}{ Taichung ${ }^{u}$} \\
\hline & & $n$ & Freq & & & & & 1996-2002 & 2009-2011 & 2012-2013 & 1996-2002 & 2009-2011 & 2012-2013 \\
\hline $\mathrm{A}$ & 162 & 73 & 0.55 & 0.94 & 0.97 & 5.63 & 2.11 & 23 & 8 & 131 & 13 & 0 & 15 \\
\hline $\mathrm{C}$ & 367 & 43 & 0.88 & 0.72 & 0.79 & 3.00 & 1.37 & 5 & 15 & 347 & 5 & 1 & 31 \\
\hline $\mathrm{D}$ & 19 & 17 & 0.11 & 0.98 & 0.98 & 4.94 & 3.90 & 0 & 7 & 12 & 0 & 0 & 0 \\
\hline Total & 637 & 154 & 0.76 & 0.90 & 0.94 & 3.81 & 2.47 & 28 & 34 & 575 & 18 & 1 & 56 \\
\hline
\end{tabular}

${ }^{\mathrm{t}}$ Number $(n)$ and frequency (Freq) of unique haplotypes.

" Data are numbers of isolates from three different times. Overall Taiwan $=637$ isolates from all around Taiwan. Taichung $=75$ isolates from Taichung.

v Genetic cluster.

w Number of isolates.

x Genotypic diversity (GD) (clone-corrected)

y Mean number of alleles per locus.

$\mathrm{z}$ Allelic richness averaged over loci.

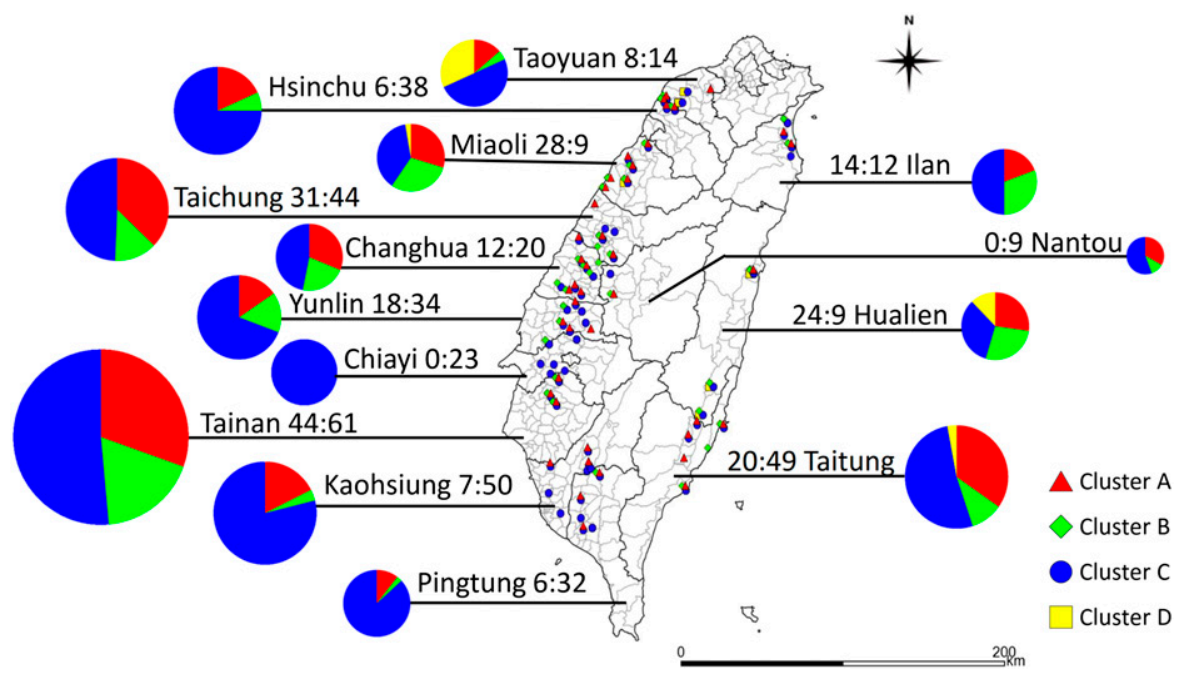

Fig. 2. Geographical distribution of different genetic groups and mating types of Fusarium fujikuroi isolates in Taiwan. Isolates in genetic clusters A, B, C, and D are represented by different colors. Sizes of different pie charts are proportional to the numbers of isolates collected from the cities or counties. Each pie chart shows the ratio of different genetic clusters in the collection area. Numbers labeled by each pie chart indicate the number of MAT1-1 isolates/number of MAT1-2 isolates in that city or county. 
the mating type ratio deviated significantly from $1: 1$ in the overall Taiwan population, clusters $\mathrm{B}$ and $\mathrm{C}$, and 8 of the 14 subpopulations in different cities or counties (Table 3 ). The effective population numbers in clusters B and C were reduced to approximately $60 \%$ of the actual counts. The biased mating type ratios in six cities or counties also largely reduced the effective population numbers to 0\% (Chiayi and Nantou), 58\% (Kaohsiung), and approximately $70 \%$ (Hsinchu, Miaoli, and Pingtung) (Table 3). The $I_{A}$ and $\bar{r}_{d}$ tests showed that, among the four clusters, only cluster B was at linkage equilibrium. On the regional scale, none of the subpopulations in individual cities or counties were at linkage equilibrium (Table 3).

VCG. Analysis of $21 \mathrm{~F}$. fujikuroi isolates revealed $16 \mathrm{VCG}$, including 11 VCG among 13 diverse isolates from different areas of Taiwan and 6 VCG among 8 local isolates from Taichung. No heterokaryon self-incompatible isolates were observed. From the eight Taichung isolates, the five isolates from 2000 and the three isolates from 2012 were associated with four and three VCG, respectively (VCG test set II). The results suggested high VCG diversity in both early and recent $F$. fujikuroi populations in Taiwan. No relationship was found among mating type, VCG, and SSR genetic cluster.

Virulence of representative $F$. fujikuroi isolates on different rice varieties. The results of virulence test I ( $24 \mathrm{~F}$. fujikuroi isolates inoculated on eight rice varieties) are shown in Figure 3 and Supplementary Fig. S2, and the results of virulence test II (60 isolates on three rice varieties) are shown in Figure 4 and Table 4. All tested isolates were pathogenic to rice. Variations were observed among diverse isolates. The isolates of genetic clusters $\mathrm{A}, \mathrm{B}$, and $\mathrm{C}$ showed significantly higher degrees of virulence than those of cluster D in both virulence tests (Figs. 3 and 4). In general, highly virulent isolates caused greater disease severity and isolates with low virulence caused less disease severity on all selected rice varieties, which suggested that none of the rice varieties displayed specific resistance to particular F. fujikuroi isolates. Among the eight rice varieties, Budda showed significantly greater resistance; IR78581-12-3-2-2, and TN11 showed moderate resistance; and Koshihikari, Toyonishiki, and TNG71 showed greater susceptibility to F. fujikuroi isolates (Fig. 3). The results conformed to the observations of Dr. M.-H. Lai in previous screening tests of rice germplasm in Taiwan (M.-H. Lai, unpublished data). In virulence test II, three principal rice varieties from different times showed consistent levels of resistance or susceptibility to $F$. fujikuroi isolates collected from early years (1998 and 2002) and recent years (2012), regardless of the use of the isolates from all of Taiwan or from Taichung for the analysis (Table 4). For both early and recent isolates, the levels of resistance (from high to low) were TN11 > TNG67 = TNG71 (Fig. 4). Analysis of virulence of the 35 haplotypes on TNG71 showed that haplotypes H023, H039, and H055 were highly virulent, and H002, H119, H120, H132, $\mathrm{H} 134$, and H135 (all belonging to cluster D) were less virulent (Supplementary Fig. S3).

Fungicide sensitivity. The sensitivity of 60 representative F. fujikuroi isolates to prochloraz and tebuconazole are summarized in Table 5. Significant differences were observed between cluster D and clusters $\mathrm{A}, \mathrm{B}$, and $\mathrm{C}$ for inhibitory effects of prochloraz and tebuconazole on both spore germination and mycelial growth. Compared with the isolates of clusters A, B, and C, those of cluster $\mathrm{D}$ were significantly more sensitive to prochloraz (lower $\mathrm{EC}_{50}$ ) and more resistant to tebuconazole (higher $\mathrm{EC}_{50}$ ). The differences among the 35 haplotypes in fungicide sensitivity are shown in Supplementary Fig. S3.

Significant differences were also revealed between early isolates (from 1998 and 2000) and recent isolates (from 2012), particularly for prochloraz (Table 5). Because cluster D and clusters A, B, and C had significantly different levels of sensitivity to prochloraz and tebuconazole and cluster D was only found in recent years, we used the datasets with and without the isolates of cluster D for statistical analyses (Table 5, "cluster D included" and "cluster D excluded"). Similar results were obtained from both datasets. Recent isolates showed an approximately 2-fold and approximately 4.5-fold greater $\mathrm{EC}_{50}$ of prochloraz on spore germination and mycelial growth, respectively, than the early isolates. For tebuconazole, recent isolates showed a lower $\mathrm{EC}_{50}$ (approximately 0.5-fold) on spore germination and a lower $\mathrm{EC}_{50}$ (approximately 0.7 -fold) on mycelial growth than early isolates. The results suggested that the recent

TABLE 3. Mating type frequencies, effective population numbers, and multilocus linkage disequilibrium tests for the four genetic clusters and 14 geographical subpopulations of Fusarium fujikuroi in Taiwan ${ }^{\mathrm{y}}$

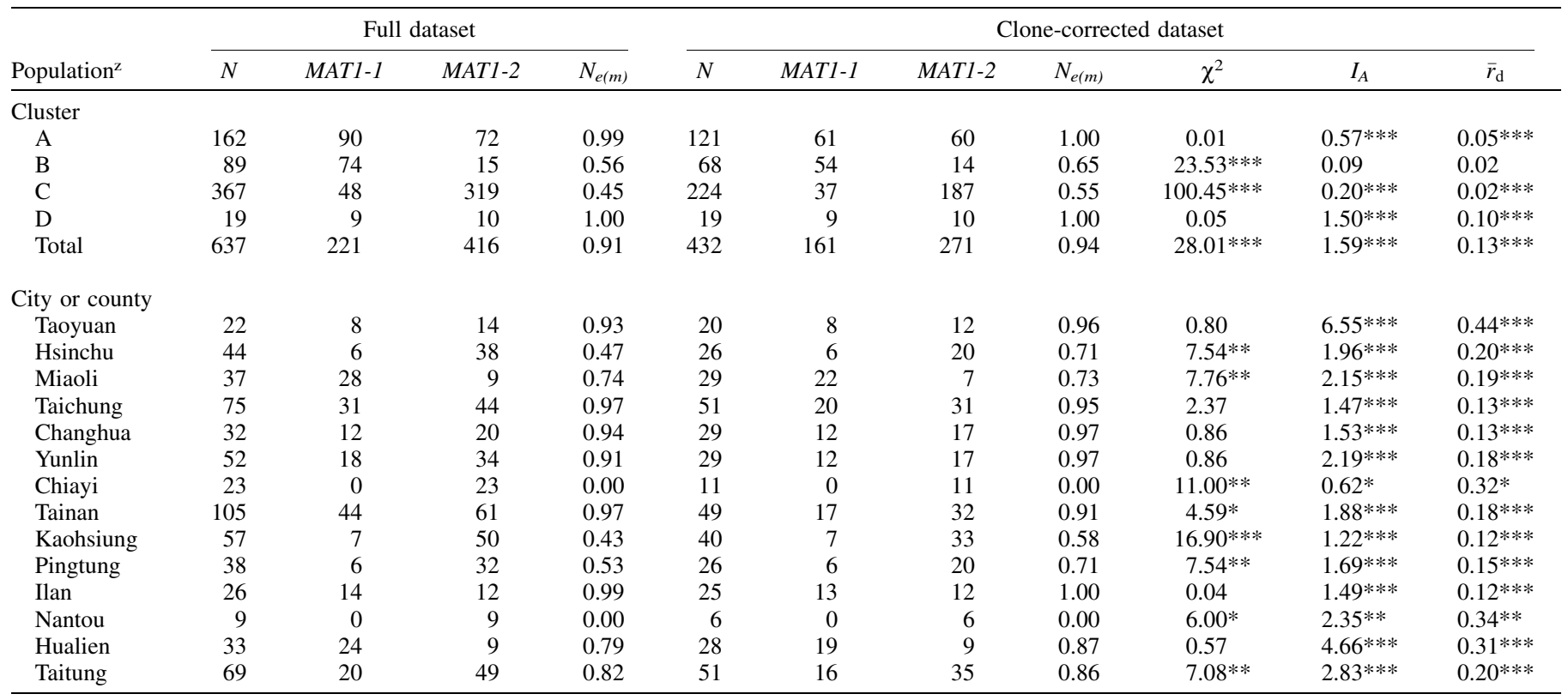

y $N=$ total number of isolates; $M A T 1-1$ and $M A T 1-2=$ number of $M A T 1-1$ and $M A T 1-2$ isolates, respectively; $N_{e(m)}=$ effective population number, calculated by the number of MAT1-1 and MAT1-2 isolates and expressed as the proportion of the actual count.; $\chi^{2}=$ goodness of fit test, based on a $1: 1$ mating type ratio with

1 degree of freedom; $I_{A}=$ index of association, as calculated in Multilocus v3.1 with 1,000 randomizations; and $\bar{r}_{\mathrm{d}}=$ multilocus association, as calculated in Multilocus v3.1 with 1,000 randomizations. Asterisks: * indicates $0.05>P>0.01$; ** indicates $0.01>P>0.001$; and *** indicates $P<0.001$.

${ }^{\mathrm{z}}$ Genetic cluster or geographical subpopulation. 
F. fujikuroi population is more resistant to prochloraz and slightly more sensitive to tebuconazole.

\section{DISCUSSION}

Bakanae disease is one of the newly emerging problems of rice production in some areas of the world, including Taiwan. Previous studies of its causal agent, F. fujikuroi, have focused on the taxonomy, molecular identification, pathogenicity, and production and toxicity of secondary metabolites such as $\mathrm{GA}_{3}$, fusaric acid, moniliformin, and fumonisin (Avalos et al. 2007; Desjardins et al. 1997, 2000; Harvey et al. 2001; Hsuan et al. 2011; Niehaus et al. 2014; Singh and Sunder 2012). Some recent works investigated the development of efficient and reliable methods of inoculation and disease evaluation (Amatulli et al. 2012; Hsu et al. 2013b; Hwang et al. 2013; Kim et al. 2014). Although mating type, VCG, AFLP, and UP-PCR analyses were performed to investigate $F$. fujikuroi populations (Carter et al. 2008; Cumagun et al. 2011; Mohd Zainudin et al. 2008a; Puhalla and Spieth 1985; Sunder and Satyavir 1998), thus far, codominant molecular markers have not been developed. In addition, no study has been conducted to explore temporal changes in the pathogenicity and fungicide resistance in an $F$. fujikuroi population.

In this study, a set of 16 SSR markers was newly developed for better understanding of the molecular genetic diversity and genetic structure of F. fujikuroi in Taiwan. These markers are locus-specific, easy to amplify, and highly polymorphic. For all 16 SSR loci in each of the 637 isolates, only one allele per locus was detected, which suggests that $F$. fujikuroi is haploid in the infected rice seeds and seedlings. The F. fujikuroi population in Taiwan was classified into four highly differentiated genetic clusters and 154 haplotypes.
Compared with cluster B, cluster A possesses higher genetic diversity and clusters $\mathrm{A}$ and $\mathrm{C}$ were geographically and temporally more widespread (isolates of cluster B were from later than 2009 and mostly sampled from areas north of Tainan). Because the alleles in cluster B are subsets of those in clusters A and C, cluster B likely derived from clusters A or C. Cluster D, consisting of only 19 isolates located in four cities or counties, featured high genetic diversity. In cluster D, the allelic compositions at the 16 SSR loci did not resemble those of clusters A, B, and C; therefore, the isolates of cluster D could have been introduced from unknown sources genetically distinct from clusters A, B, and C. In support of the genetic data, we observed that cluster D had significantly different levels of virulence (Fig. 4) and fungicide sensitivity (Table 5) than the other three clusters. Among the diverse haplotypes revealed in the study, haplotypes H039, H064, H065, and H145 were predominant and widespread in Taiwan. The observed predominance may be associated with fitness advantages or human activities (e.g., preferred sources of seeds or seedlings). Isolates of H039, H064, H065, and H145 showed moderate (to high) levels of virulence and prochloraz tolerance but the virulence and prochloraz tolerance of the four haplotypes were not significantly superior to those of the rest of the tested haplotypes. It is speculated that the clonal expansion may also be related to human activities or the ability of the pathogen to tolerate other stresses and adapt to diverse environments.

Genetic variations in the $F$. fujikuroi population in Taiwan are likely associated with sexual reproduction. Good numbers of MAT1-1 and MAT1-2 isolates could be sampled from all around Taiwan, which suggests the possibility of isolates of opposite mating types mating with each other. In support of this finding, Yu and Sun (1976) observed that, by the time of heading stage, the perithecia of $F$. fujikuroi began to appear on the basal portion,

TABLE 4. Results of virulence test II on principal rice varieties

\begin{tabular}{|c|c|c|c|c|c|}
\hline \multirow[b]{2}{*}{ Variety } & \multirow[b]{2}{*}{ Years $^{\mathrm{z}}$} & \multicolumn{2}{|c|}{ Overall Taiwan (60 isolates $)^{\mathrm{y}}$} & \multicolumn{2}{|c|}{ Taichung (12 isolates) $)^{\mathrm{y}}$} \\
\hline & & Early (8) & Recent (52) & Early (8) & Recent (4) \\
\hline TNG67 & 1979-1998 & $0.28 \pm 0.03 \mathrm{a}$ & $0.30 \pm 0.01 \mathrm{a}$ & $0.28 \pm 0.03 \mathrm{ab}$ & $0.26 \pm 0.03 \mathrm{a}$ \\
\hline TNG71 & $2003-2010$ & $0.35 \pm 0.03 \mathrm{a}$ & $0.31 \pm 0.01 \mathrm{a}$ & $0.35 \pm 0.03 \mathrm{a}$ & $0.26 \pm 0.03 a$ \\
\hline TN11 & 2005-now & $0.18 \pm 0.03 b$ & $0.18 \pm 0.01 b$ & $0.19 \pm 0.03 b$ & $0.11 \pm 0.03 b$ \\
\hline
\end{tabular}

${ }^{\mathrm{x}}$ Early and recent isolates were evaluated for virulence on Tainung 67 (TNG67), TNG71 (Tainung 71), and Tainan 11 (TN11), the principal varieties at different times. Data are least squares mean \pm standard error. Within each column, data with the same letter are considered not significantly different from each other, by the Tukey-Kramer test at $P<0.05$.

y Early $=$ isolates from 1998 and 2002, Recent $=$ isolates from 2012, and numbers in parentheses indicate number of isolates in each time period.

z Principal variety during these years.

TABLE 5. Inhibitory effects of prochloraz and tebuconazole on spore germination and mycelial growth of Fusarium fujikuroi in four genetic clusters and from different collection years

\begin{tabular}{|c|c|c|c|c|c|}
\hline \multirow[b]{3}{*}{ Genetic cluster, collection year ${ }^{\mathrm{y}}$} & \multirow[b]{3}{*}{ Number of isolates } & \multicolumn{4}{|c|}{ Inhibition of ${ }^{x}$} \\
\hline & & \multicolumn{2}{|c|}{ Spore germination $\left(\mathrm{EC}_{50}, \mathrm{mg} / \mathrm{liter}\right)$} & \multicolumn{2}{|c|}{ Mycelial growth $\left(\mathrm{EC}_{50}, \mathrm{mg} / \mathrm{liter}\right)$} \\
\hline & & Prochloraz & Tebuconazole & Prochloraz & Tebuconazole \\
\hline Cluster A & 18 & $18.28 \pm 1.91 \mathrm{a}$ & $1.59 \pm 0.10 b$ & $3.54 \pm 0.85 b$ & $0.22 \pm 0.03 b$ \\
\hline Cluster B & 14 & $20.79 \pm 2.17 \mathrm{a}$ & $0.61 \pm 0.11 \mathrm{~d}$ & $10.32 \pm 0.97 \mathrm{a}$ & $0.22 \pm 0.03 \mathrm{~b}$ \\
\hline Cluster C & 20 & $21.87 \pm 1.81 \mathrm{a}$ & $1.00 \pm 0.09 \mathrm{c}$ & $7.31 \pm 0.80 \mathrm{a}$ & $0.18 \pm 0.03 b$ \\
\hline Cluster D & 8 & $0.45 \pm 2.87 \mathrm{~b}$ & $2.16 \pm 0.15 \mathrm{a}$ & $0.74 \pm 1.28 \mathrm{~b}$ & $0.74 \pm 0.04 \mathrm{a}$ \\
\hline Early (cluster D included) ${ }^{\mathrm{z}}$ & 8 & $10.25 \pm 2.25$ & $1.96 \pm 0.18$ & $1.63 \pm 1.45$ & $0.29 \pm 0.06$ \\
\hline Recent (cluster D included) & 52 & $25.05 \pm 2.83 * * *$ & $0.97 \pm 0.22 * * *$ & $6.68 \pm 0.56 * * *$ & $0.27 \pm 0.02$ \\
\hline Early (cluster D excluded)z & 8 & $10.25 \pm 2.88$ & $1.96 \pm 0.15$ & $1.63 \pm 1.42$ & $0.29 \pm 0.04$ \\
\hline Recent (cluster D excluded) & 43 & $22.17 \pm 1.23^{* * *}$ & $0.94 \pm 0.07 * * *$ & $7.75 \pm 0.60 * * *$ & $0.19 \pm 0.01$ \\
\hline Early from Taichung & 8 & $10.25 \pm 2.23$ & $1.96 \pm 0.17$ & $1.63 \pm 0.55$ & $0.29 \pm 0.05$ \\
\hline Recent from Taichung & 4 & $25.04 \pm 2.83 * * *$ & $0.97 \pm 0.22 * * *$ & $6.73 \pm 0.69 * * *$ & $0.20 \pm 0.07$ \\
\hline
\end{tabular}

${ }^{x} \mathrm{EC}_{50}=$ the effective dose that causes $50 \%$ inhibition of spore germination or mycelial growth. Data are mean \pm standard error of the EC 50 values.

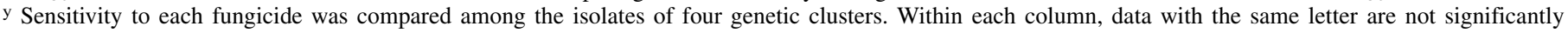
different from each other, by the Tukey-Kramer test at $P<0.05$.

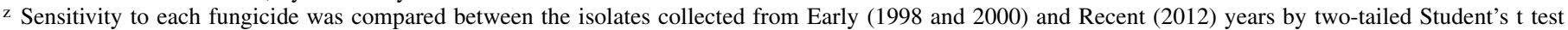
(data in each column were compared). * indicates $0.05>P>0.01$, ** indicates $0.01>P>0.001$, and $* * *$ indicates $P<0.001$. 
moving gradually to the upper portion of the diseased rice stem. A year-round field survey in Taichung with the Hirst automatic volumetric spore trap also showed that the ascospores were discharged into the air from heading through harvesting stage (Sun 1975; Yu and Sun 1976). In this study, high VCG diversity was detected in a set of 13 diverse isolates from different areas of Taiwan and 8 local isolates from Taichung, which suggests that $F$. fujikuroi has long existed in Taiwan and has been sexually reproduced in the field. In fungi, isolates belonging to the same VCG are vegetatively compatible and are thought to share common alleles at the vegetative

\section{Cluster A}

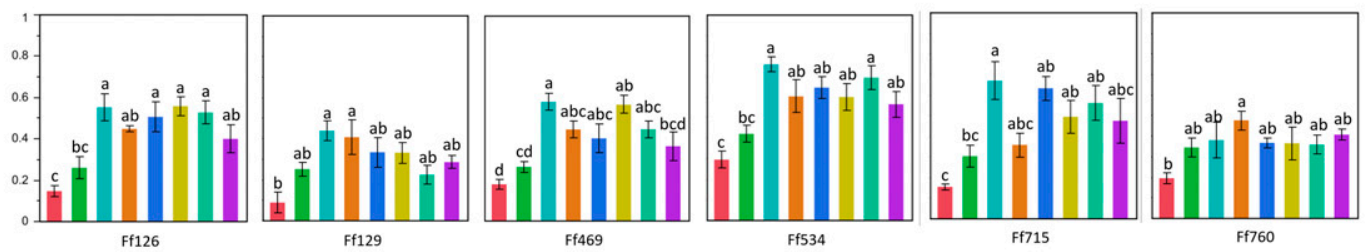

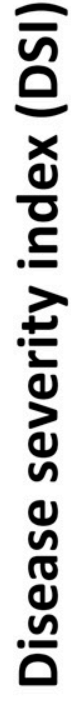

\section{Cluster B}

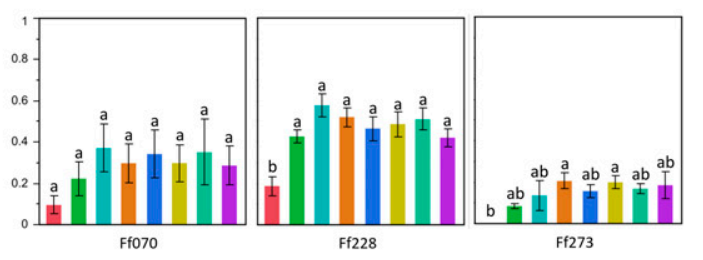

Cluster D

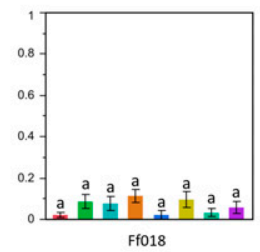

\section{Rice variety}

- Budda Kaohsiung 139 (KH139)

- IR78581-12-3-2-2 ainung 71 (TNG71)

- Toyonishiki Taikeng 16 (TK16)

- Koshihikari $\quad$ Tainan 11 (TN11)

\section{Cluster C}
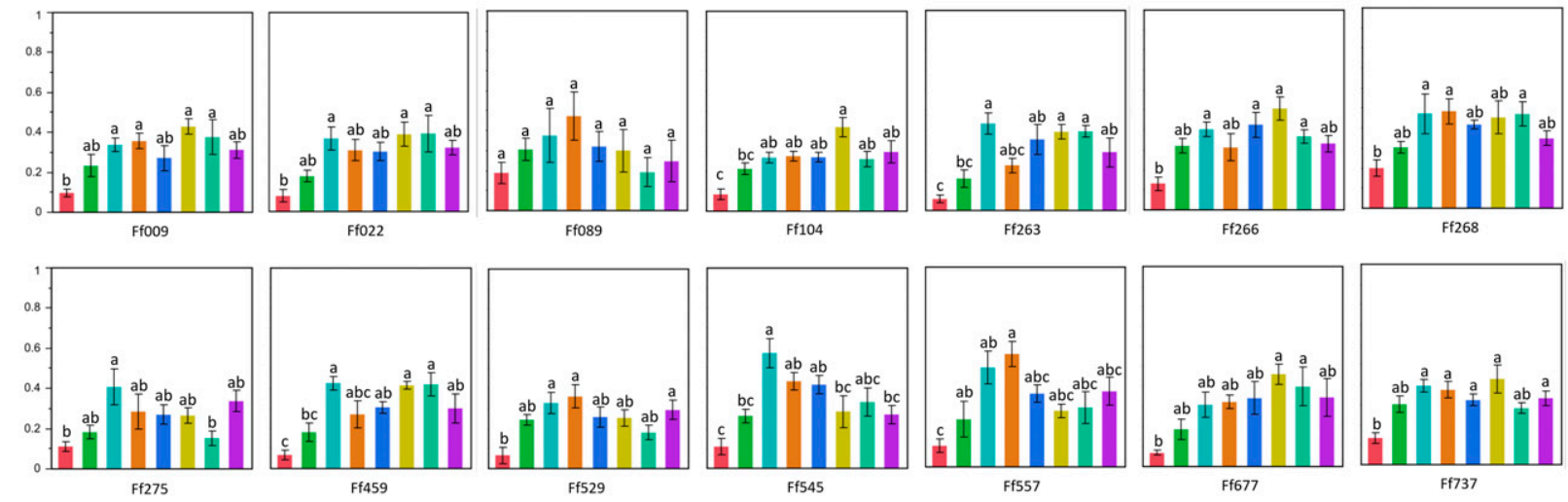

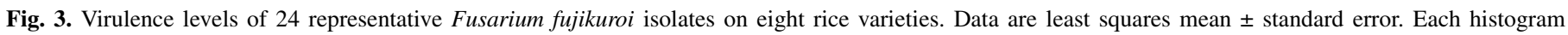

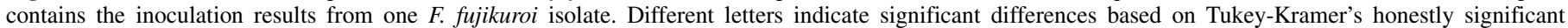
difference test at $P<0.05$. $\mathrm{X}$-axis $=$ rice variety and $\mathrm{Y}$-axis $=$ disease severity index.
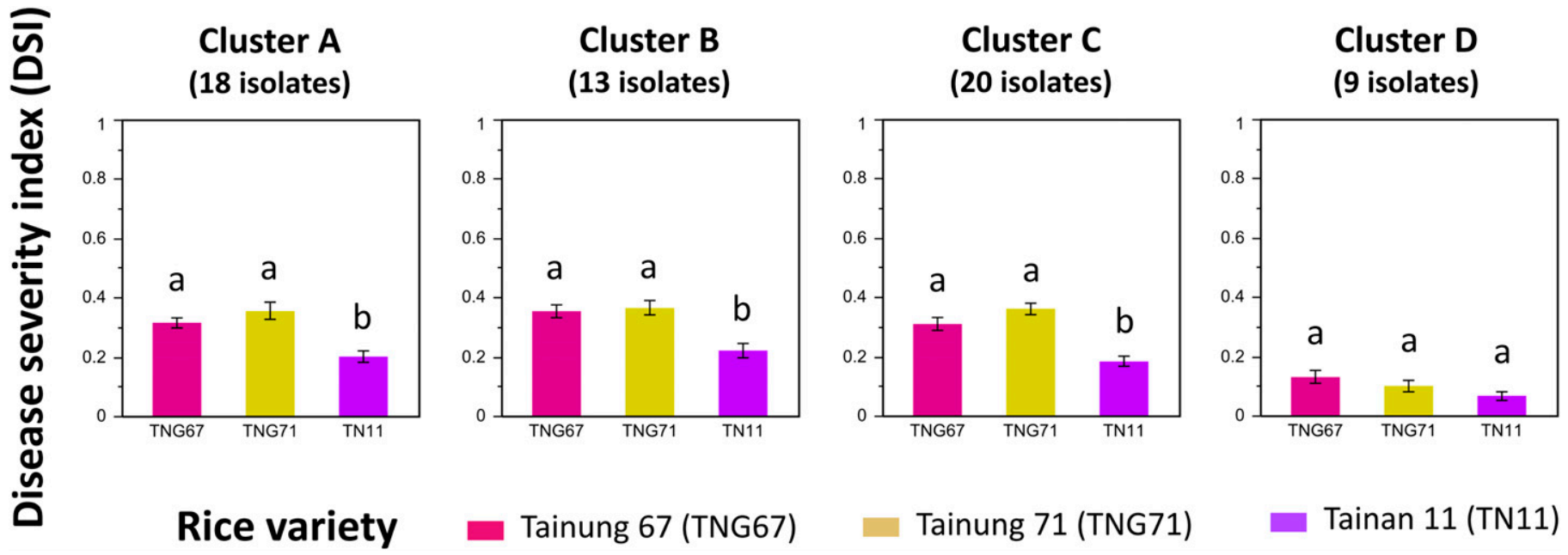

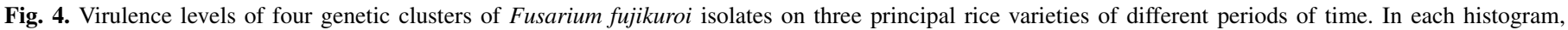
different letters indicate significant differences based on Tukey-Kramer's honestly significant difference test at $P<0.05$. 
incompatibility (vic) loci (Leslie 1993). Low degree of VCG diversity usually implies the predominance of clonal reproduction in a population. In contrast, mating and meiotic recombinations occurring in a sexual population can result in new variability in the vic loci and, thus, an increased level of VCG diversity. In F. fujikuroi, high VCG diversity was revealed in populations in China and Taiwan (Puhalla and Spieth 1985), India (Sunder and Satyavir 1998), and Malaysia and Indonesia (Mohd Zainudin et al. 2008a), whereas the newly established F. fujikuroi population in California in the United States exhibited low VCG diversity (Carter et al. 2008). Our results in this study agree with the study by Puhalla and Spieth (1985), in which four isolates from Taiwan were assigned to four different VCG.

Although the data supported sexual reproduction, the overall Taiwan population; genetic clusters A, C, and D; and all 14 regional subpopulations were not at linkage equilibrium, which indicates that the individuals did not mate randomly. The high levels of differentiation between genetic clusters A, B, C, and D also suggested that sexual reproduction events did not occur frequently between clusters. Three hypotheses might explain the nonrandom mating observed in F. fujikuroi: (i) reproductive constraints due to the presence of female-sterile and poorly fertile individuals in the population, (ii) the population responding to strong selection by fungicides and other control measures, and (iii) limited movement and infection efficiency of propagules in the field. In field populations of heterothallic ascomycetes, the extent of sexual reproduction can be affected by the ratio of MAT1-1/MAT1-2 and also the male, female, or hermaphroditic nature of individual members of the population. The loss of female fertility, perhaps due to mutation or selection, leads to the emergence of female-sterile strains that would be at a selective disadvantage during sexual reproduction and a selective advantage during vegetative propagation (Leslie and Klein 1996). Previous studies of different species in GFSC, including F. verticillioides (MP-A), F. proliferatum (MP-D), $F$. subglutinans f. sp. pini (MP-E), and F. thapsinum (MP-F), showed that female-sterile mutants may comprise $>50 \%$ of the population (Britz et al. 1998; Leslie and Klein 1996; Venturini et al. 2011). In this study, biased mating type ratios and largely reduced effective population numbers were detected in clusters B and C and six regional subpopulations, which indicates some level of inbreeding. The mating behavior of $F$. fujikuroi is largely unknown and worth further exploration. In the future, sexual crosses with standard tester strains should be done to determine the inbreeding effective population number $\left(N_{e(f)}\right)$ from the proportion of femalesterile and hermaphroditic strains within the population.

Nonrandom mating can also occur when mates are physically inaccessible. In Taiwan, rice seedlings are raised in the nursery and then mechanically transferred from seedling trays into the paddy field. Because seed treatment is a commonly adopted control measure, only the genotypes resistant or tolerant to fungicides will survive and have the chance to mate. In the nursery station, diseased seedlings can be identified by visual inspection and discarded. Therefore, when good practices are followed, a small number of diseased seedlings would be sparsely distributed in the field. In addition, although $F$. fujikuroi can survive in plant debris for more than 4 months (Huang and Chu 2009; Singh and Sunder 2012), enough inoculum is required for successful infection. Inoculum density $<1.61 \times 10^{2}$ propagules/g of soil was unable to cause disease on 20-day-old rice seedlings (Yu and Sun 1977). Moreover, F. fujikuroi conidia or ascospores formed on the infected stem may disperse only to the panicles and seeds of neighboring plants. The active discharge distance of $F$. fujikuroi ascospores in still air was $28 \mathrm{~cm}$ (Yu and Sun 1976). In the paddy field, long-distance transmission by water is possible but the aboveground vegetative parts are not sites of infection (Sun 1975). The significant IBD pattern in all of Taiwan and the four genetic clusters also suggested geographically restricted gene flow and low dissemination ability of $F$. fujikuroi. The small IBD slopes may be associated with some interfering effects from the movement of contaminated rice seeds by humans.

Resistance to particular $F$. fujikuroi isolates, an indication of qualitative or vertical resistance controlled by major resistance $(R)$ genes, was not observed in the rice varieties tested in this study. Little is known about rice resistance to bakanae disease. Three quantitative trait loci have been mapped to rice chromosomes 1 and 10 (Hur et al. 2015; Yang et al. 2006) but the causal genes have yet to be cloned. It is also unclear whether the interactions between rice and $F$. fujikuroi involve the typical host $R$ gene-pathogen avirulence gene relationship. The elongation, etiolation, and stunting symptoms of bakanae disease have been found associated with the physiological responses of rice to $F$. fujikuroi-produced $\mathrm{GA}_{3}$ and fusaric acid (Ou 1985; Singh and Sunder 2012). However, in different rice genotypes, the degrees of bakanae resistance are not necessarily associated with levels of sensitivity to $\mathrm{GA}_{3}$ (Kim et al. 2014). In this study, among the representative isolates from different genetic clusters, geographical areas, and collection years, we found differential levels of virulence but did not observe a clear pattern of specific variety $\times$ isolate interaction. This finding has important implications for resistance breeding: instead of using multiple isolates for inoculation, a single highly virulent isolate of $F$. fujikuroi might be appropriate for preliminary screening of rice accessions for bakanae resistance. In the future, more rice varieties should be tested with diverse isolates to examine the validity of the proposed principle.

$F$. fujikuroi may not evolve quickly enough to overcome host resistance within several years. In virulence test I, Budda rice exhibited superior resistance to all 24 of the representative isolates; therefore, it could be a good resistance donor in breeding programs. In virulence tests I and II, TN11 showed a higher degree of resistance than the other local popular commercial varieties (TNG67, TNG71, KH139, TN11, and TK16), and the resistance was consistently effective with both early and recent isolates (Table 4). In Taiwan, TN11 was released in 2004 and has been the most predominant variety (accounting for approximately $50 \%$ of the rice cultivation area) since 2006 (Lo 2011). Of note, TN11 initially had a strong resistance to blast but the resistance was soon overcome in 2006 (Chen et al. 2009). Although the mechanisms underlying the relatively more durable resistance against bakanae disease in TN11 remain to be resolved, the results suggest two different host-pathogen coevolutionary patterns in rice-F. fujikuroi and rice-Magnaporthe oryzae pathosystems. This does not exclude the possibility of some shared components in the two pathosystems. A recent study showed that, upon inoculation with $F$. fujikuroi, upregulation of phytoalexins was detected in a resistant cultivar (Siciliano et al. 2015).

The F. fujikuroi population in Taiwan has evolved resistance to prochloraz. For rice seed treatment in Taiwan, prochloraz has been the most widely used fungicide over the past 30 years (Huang and Chu 2009), and tebuconazole has been increasingly adopted by farmers since 2009 to 2010 . The fungicide sensitivity tests of early and recent isolates suggested that the effectiveness of prochloraz has changed during the years. The early population was more sensitive to prochloraz and the recent population was more tolerant. A few highly resistant isolates (Ff070, Ff426, and Ff545) were also detected in the recent population. Considering the genetic diversity of the F. fujikuroi population and the lack of any highly virulent clones widespread in Taiwan, the outbreak of bakanae disease in eastern Taiwan during 2009 was likely due to the emergence of prochloraz-tolerant and -resistant isolates. In addition, although both prochloraz and tebuconazole belong to the azole fungicides, known to interfere with ergosterol biosynthesis at the C-14 $\alpha$ demethylation step in fungi (Brent 1995), the increased prochloraz resistance was not accompanied by increased resistance to tebuconazole (Table 5) (tebuconazole-resistant isolates were only found in cluster D). The lack of cross-resistance between different azole fungicides (particularly prochloraz, tebuconazole, triflumizole, and fluquinconazole) has been reported in field isolates of Mycosphaerella graminicola in France (Leroux et al. 2007). In 
South Korea, F. fujikuroi isolates showing resistance to both or either one of prochloraz and tebuconazole were observed (Lee et al. 2010). It would be intriguing to determine the mechanisms involved in the emerging prochloraz and tebuconazole resistance in the recent $F$. fujikuroi population in Taiwan.

Bakanae is an important seedborne disease; therefore, the pathogen population is subject to strong selection of seed treatment fungicides. Similar to the case in Taiwan, long-term and excessive use of prochloraz has made the fungicide ineffective for $F$. fujikuroi control in South Korea (Kim et al. 2010). The development of increased resistance to azole fungicides has also been reported in other fungi (Chen et al. 2012; Spolti et al. 2012). In Taiwan, tebuconazole has been widely used to treat seeds for 5 to 6 years. Although tebuconazole is still effective, a few resistant isolates were already present in the population. To minimize the risk of fungicide resistance, farmers are advised to use tebuconazole in rotation with alternative fungicides with different modes of action. Seeds treated with $62.5 \%$ cyprodinil + fludioxonil WG $1,000 \times$, $20 \%$ pefurazorate WP $1,000 \times, 40 \%$ benomyl + thiram WP $1,000 \times$, or $80 \%$ thiophanate + thiram mixture WP $800 \times$ provided satisfactory control of bakanae disease (Huang and Chu 2009).

\section{ACKNOWLEDGMENTS}

We thank C.-C. Hsu, S.-H. Li, and S.-Y. Chen for their help with F. fujikuroi culture, inoculation, and disease evaluation; and J.-F. Chen, M.-T. Lai, and J.-Y. Wang for help with sample collection and isolation. This work was funded by the National Taiwan University, Taiwan Agricultural Research Institute, and Bureau of Animal and Plant Inspection and Quarantine, Council of Agriculture, Taiwan.

\section{LITERATURE CITED}

Abdel-Mawgood, A. L. 2012. DNA based techniques for studying genetic diversity. Pages 95-122 in: Genetic Diversity in Microorganisms. M. Caliskan, ed. InTech, Rijeka, Croatia.

Agapow, P.-M., and Burt, A. 2001. Indices of multilocus linkage disequilibrium. Mol. Ecol. Notes 1:101-102.

Amatulli, M. T., Spadaro, D., Gullino, M. L., and Garibaldi, A. 2010. Molecular identification of Fusarium spp. associated with bakanae disease of rice in Italy and assessment of their pathogenicity. Plant Pathol. 59:839-844.

Amatulli, M. T., Spadaro, D., Gullino, M. L., and Garibaldi, A. 2012. Conventional and real-time PCR for the identification of Fusarium fujikuroi and Fusarium proliferatum from diseased rice tissues and seeds. Eur. J. Plant Pathol. 134:401-408.

Avalos, J., Cerdá-Olmedo, E., Reyes, F., and Barrero, A. F. 2007. Gibberellins and other metabolites of Fusarium fujikuroi and related fungi. Curr. Org. Chem. 11:721-737.

Benson, G. 1999. Tandem repeats finder: A program to analyze DNA sequences. Nucleic Acids Res. 27:573-580.

Brent, K. J. 1995. Fungicide Resistance in Crop Pathogens: How Can it be Managed? FRAC Monograph No. 1. Global Crop Protection Federation, Brussels, Belgium.

Britz, H., Wingfield, M. J., Coutinho, T. A., Marasas, W. F. O., and Leslie, J. F. 1998. Female fertility and mating type distribution in a South African population of Fusarium subglutinans f. sp. pini. Appl. Environ. Microbiol. 64:2094-2095

Brown, A. H. D., Feldman, M. W., and Nevo, E. 1980. Multilocus structure of natural populations of Hordeum spontaneum. Genetics 96:523-536.

Brownstein, M. J., Carpten, J. D., and Smith, J. R. 1996. Modulation of nontemplated nucleotide addition by Taq DNA polymerase: Primer modifications that facilitate genotyping. Biotechniques 20:1004-1010.

Carter, L. L. A., Leslie, J. F., and Webster, R. K. 2008. Population structure of Fusarium fujikuroi from California rice and water grass. Phytopathology 98:992-998.

Chang, Y.-C. 2003. Rice bakanae disease. Pages 256-257 in: Compendium of Rice Diseases and Pests. Bureau of Animal and Plant Health Inspection and Quarantine, Taipei City, Taiwan. In Chinese.

Chen, F. P., Fan, J. R., Zhou, T., Liu, X. L., Liu, J. L., and Schnabel, G. 2012. Baseline sensitivity of Monilinia fructicola from China to the DMI fungicide SYP-Z048 and analysis of DMI-resistant mutants. Plant Dis. 96: 416-422.

Chen, L.-C., Huang, S.-H., and Cheng, C.-H. 2009. Review of the screening test for rice varietal resistance to major diseases and insect pests in Taiwan. Pages
83-103 in: Proc. Symp. Achiev. Perspect. Rice Prot. Taiwan. H.-F. Ni and H.-R. Yang, eds. Chiayi Agricultural Experiment Branch, Taiwan Agricultural Research Institute, Chiayi, Taiwan. (In Chinese with English abstract)

Chung, C.-L., Huang, S.-Y., Huang, Y.-C., Tzean, S.-S., Ann, P.-J., Tsai, J.-N., Yang, C.-C., Lee, H.-H., Huang, T.-W., Huang, H.-Y., Chang, T.-T., Lee, H.-L., and Liou, R.-F. 2015. The genetic structure of Phellinus noxius and dissemination pattern of brown root rot disease in Taiwan. PLoS One 10: $\mathrm{e} 0139445$.

Correll, J. C., Klittich, C., and Leslie, J. F. 1987. Nitrate nonutilizing mutants of Fusarium oxysporum and their use in vegetative compatibility tests. Phytopathology 77:1640-1646.

Cumagun, C. J. R., Arcillas, E., and Gergon, E. 2011. UP-PCR analysis of the seed borne pathogen Fusarium fujikuroi causing bakanae disease in rice. Int. J. Agric. Biol. 13:1029-1032.

Desjardins, A. E., Manandhar, H. K., Plattner, R. D., Manandhar, G. G., Poling, S. M., and Maragos, C. M. 2000. Fusarium species from Nepalese rice and production of mycotoxins and gibberellic acid by selected species. Appl. Environ. Microbiol. 66:1020-1025.

Desjardins, A. E., Plattner, R. D., and Nelson, P. E. 1997. Production of fumonisin b (inf1) and moniliformin by Gibberella fujikuroi from rice from various geographic areas. Appl. Environ. Microbiol. 63: $1838-1842$

Doyle, J. J., and Doyle, J. L. 1987. A rapid DNA isolation procedure for small quantities of fresh leaf tissue. Phytochem. Bull. 19:11-15.

Elmer, W. H., Summerell, B. A., Burgess, L. W., and Nigh, E. L., Jr. 1999. Vegetative compatibility groups in Fusarium proliferatum from asparagus in Australia. Mycologia 91:650-654.

Evanno, G., Regnaut, S., and Goudet, J. 2005. Detecting the number of clusters of individuals using the software STRUCTURE: A simulation study. Mol. Ecol. 14:2611-2620

Goudet, J. 2001. FSTAT, a program to estimate and test gene diversities and fixation indices (version 2.9.3). Updated from Goudet 1995. Online publication. http://www2.unil.ch/popgen/softwares/fstat.htm

Gupta, A. K., Singh, Y., Jain, A. K., and Singh, D. 2014. Prevalence and incidence of bakanae disease of rice in northern India. J. AgriSearch 1:233-237.

Haq, M., Mia, M. A. T., Rabbi, M. F., and Ali, M. A. 2011. Incidence and severity of rice diseases and insect pests in relation to climate change. Pages 445-457 in: Climate Change and Food Security in South Asia. R. Lal, M. V. K. Sivakumar, S. M. A. Faiz, A. H. M. Mustafizur Rahman, and K. R. Islam, eds. Springer Netherlands, Houten.

Harvey, R. B., Edrington, T. S., Kubena, L. F., Rottinghaus, G. E., Turk, J. R., Genovese, K. J., and Nisbet, D. J. 2001. Toxicity of moniliformin from Fusarium fujikuroi culture material to growing barrows. J. Food Prot. 64: 1780-1784.

Hayden, M., Nguyen, T., Waterman, A., and Chalmers, K. 2008. MultiplexReady PCR: A new method for multiplexed SSR and SNP genotyping. BMC Genomics 9:80.

Hsu, C.-C. 2013. Fusarium fujikuroi: The development of a differential medium, establishment of the disease evaluation system and investigation of the role of soil inoculum. Master's thesis, National Chung Hsing University, Taichung, Taiwan. (In Chinese with English abstract)

Hsu, C.-C., Huang, J.-W., and Chen, C.-Y. 2013a. The cause of rice bakanae disease in Taiwan. Plant Pathol. Bull. 22:279-289. (In Chinese with English abstract)

Hsu, C.-C., Lai, M.-H., Lin, C.-C., Huang, J.-W., and Chen, C.-Y. 2013b. Standardization of the protocol for evaluating susceptibility of rice to the pathogen of bakanae disease. Plant Pathol. Bull. 22:291-299. (In Chinese with English abstract)

Hsuan, H. M., Salleh, B., and Zakaria, L. 2011. Molecular identification of Fusarium species in Gibberella fujikuroi species complex from rice, sugarcane and maize from Peninsular Malaysia. Int. J. Mol. Sci. 12: 6722-6732.

Huang, T.-C., and Chu, S.-C. 2009. The occurrence and control of rice bakanae disease in Taiwan. Pages 29-43 in: Proc. Symp. Achiev. Perspect. Rice Prot. Taiwan. H.-F. Ni and H.-R. Yang, eds. Chiayi Agricultural Experiment Branch, Taiwan Agricultural Research Institute, Chiayi, Taiwan. (In Chinese with English abstract)

Hur, Y.-J., Lee, S., Kim, T., Kwon, T., Lee, J.-H., Shin, D.-J., Park, S.-K., Hwang, U.-H., Cho, J., Yoon, Y.-N., Yeo, U.-S., Song, Y.-C., Kwak, D.-Y., Nam, M.-H., and Park, D.-S. 2015. Mapping of $q B K 1$, a major QTL for bakanae disease resistance in rice. Mol. Breed. 35:78.

Hwang, D.-J., Kang, W.-R., Hwang, D.-J., Bae, S.-C., Yun, S.-H., and Ahn, I.-P. 2013. Evaluation of bakanae disease progression caused by Fusarium fujikuroi in Oryza sativa L. J. Microbiol. 51:858-865.

Jeong, H., Lee, S., Choi, G. J., Lee, T., and Yun, S.-H. 2013. Draft genome sequence of Fusarium fujikuroi B14, the causal agent of the bakanae disease of rice. Genome Announc. 1:e00035-13.

Jombart, T. 2008. adegenet: A R package for the multivariate analysis of genetic markers. Bioinformatics 24:1403-1405. 
Khan, J. A., Jamil, F. F., and Gill, M. A. 2000. Screening of rice varieties/lines against bakanae and bacterial leaf blight (BLB). Pak. J. Phytopathol. 12: 6-11.

Kim, B.-R., Han, K.-S., Hahm, S.-S., Kwon, M.-K., and Nam, Y.-G. 2015. Occurrence of the rice bakanae disease in Chungnam province. Res. Plant Dis. $21: 154$

Kim, M.-H., Hur, Y.-J., Lee, S., Kwon, T., Hwang, U.-H., Park, S.-K., Yoon, Y.-N., Lee, J.-H., Cho, J.-H., Shin, D., Kim, T., Han, S.-I., Yeo, U.-S., Song, Y.-C., Nam, M.-H., and Park, D.-S. 2014. Large-scale screening of rice accessions to evaluate resistance to bakanae disease. J. Gen. Plant Pathol. 80:408-414.

Kim, S. H., Park, M. R., Kim, Y. C., Lee, S. W., Choi, B. R., Lee, S. W., and Kim, I. S. 2010. Degradation of prochloraz by rice bakanae disease pathogen Fusarium fujikuroi with differing sensitivity: A possible explanation for resistance mechanism. J. Korean Soc. Appl. Biol. Chem. 53:433-439.

Lee, Y.-H., Kim, S.-Y., Choi, H.-W., Lee, M.-J., Ra, D.-S., Kim, I.-S., Park, J.-W., and Lee, S.-W. 2010. Fungicide resistance of Fusarium fujikuroi isolates isolated in Korea. Korean J. Pestic. Sci. 14:427-432. (In Korean with English abstract)

Leroux, P., Albertini, C., Gautier, A., Gredt, M., and Walker, A. S. 2007. Mutations in the CYP51 gene correlated with changes in sensitivity to sterol $14 \alpha$-demethylation inhibitors in field isolates of Mycosphaerella graminicola. Pest Manage. Sci. 63:688-698.

Leslie, J. F. 1993. Fungal vegetative compatibility. Annu. Rev. Phytopathol. 31:127-150.

Leslie, J. F., and Klein, K. K. 1996. Female fertility and mating type effects on effective population size and evolution in filamentous fungi. Genetics 144: 557-567.

Leslie, J. F., and Summerell, B. A., eds. 2006. The Fusarium Laboratory Manual. Blackwell Publishing, Ames, IA.

Liu, K., and Muse, S. V. 2005. PowerMarker: An integrated analysis environment for genetic marker analysis. Bioinformatics 21:2128-2129.

Lo, J.-C. 2011. A century of agriculture: Rice research and extension in Tainan District Agricultural Research and Extension Station. Tainan Dist. Agric. Newsl. 78:30-35. (In Chinese)

Mew, T.-W., and Gonzales, P. 2002. A Handbook of Rice Seedborne Fungi. International Rice Research Institute (Laguna, Philippines) Science Publishers, Inc. (Enfield, NH)

Mirtalebi, M., and Banihashemi, Z. 2014. Genetic relationship among Fusarium oxysporum f. sp. melonis vegetative compatibility groups and their relatedness to other F. oxysporum formae speciales. J. Agric. Sci. Technol. 16:931-943.

Mohd Zainudin, N. A. I., Mohamed Sidique, S. N., and Salleh, B. 2008a. Genetic diversity of Fusarium fujikuroi isolated from bakanae disease of rice on the basis of vegetative compatibility. Pertanika J. Trop. Agric. Sci. 31:271-278.

Mohd Zainudin, N. A. I., Razak, A. A., and Salleh, B. 2008b. Bakanae disease of rice in Malaysia and Indonesia: Etiology of the causal agent based on morphological, physiological and pathogenicity characteristics. J. Plant Prot. Res. 48:475-485.

Niehaus, E. M., von Bargen, K. W., Espino, J. J., Pfannmuller, A., Humpf, H. U., and Tudzynski, B. 2014. Characterization of the fusaric acid gene cluster in Fusarium fujikuroi. Appl. Microbiol. Biotechnol. 98: 1749-1762.

Ou, S. H. 1985. Rice Diseases, 2nd ed. Commonwealth Agricultural Bureaux, Slough, UK.

Peakall, R. O. D., and Smouse, P. E. 2006. GENALEX 6: Genetic analysis in Excel. Population genetic software for teaching and research. Mol. Ecol. Notes 6:288-295.
Pritchard, J. K., Stephens, M., and Donnelly, P. 2000. Inference of population structure using multilocus genotype data. Genetics 155:945-959.

Puhalla, J. E. 1985. Classification of strains of Fusarium oxysporum on the basis of vegetative compatibility. Can. J. Bot. 63:179-183.

Puhalla, J. E., and Spieth, P. T. 1985. A comparison of heterokaryosis and vegetative incompatibility among varieties of Gibberella fujikuroi (Fusarium moniliforme). Exp. Mycol. 9:39-47.

Raymond, M., and Rousset, F. 1995. GENEPOP (version 1.2): Population genetics software for exact tests and ecumenicism. J. Hered. 86:248-249.

R Development Core Team. 2011. R: A Language and Environment for Statistical Computing. Online publication. R Foundation for Statistical Computing, Vienna. http://www.R-project.org/

Rousset, F. 2000. Genetic differentiation between individuals. J. Evol. Biol. 13:58-62.

Siciliano, I., Amaral Carneiro, G., Spadaro, D., Garibaldi, A., and Gullino, M. L. 2015. Jasmonic acid, abscisic acid, and salicylic acid are involved in the phytoalexin responses of rice to Fusarium fujikuroi, a high gibberellin producer pathogen. J. Agric. Food Chem. 63:8134-8142.

Singh, R., and Sunder, S. 2012. Foot rot and bakanae of rice: An overview. Rev. Plant Pathol. 5:565-604.

Snyder, W. C., and Sun, S. K. 1973. Heterothallism in Fusarium moniliforme. In: US-ROC Coop. Sci. Semin. Plant Root Dis. University of California, Berkeley.

Spolti, P., Jorge, B. C. d., and Del Ponte, E. M. 2012. Sensitivity of Fusarium graminearum causing head blight of wheat in Brazil to tebuconazole and metconazole fungicides. Trop. Plant Pathol. 37:419-423.

Steenkamp, E. T., Wingfield, B. D., Coutinho, T. A., Zeller, K. A., Wingfield, M. J., Marasas, W. F. O., and Leslie, J. F. 2000. PCR-based identification of MAT-1 and MAT-2 in the Gibberella fujikuroi species complex. Appl. Environ. Microbiol. 66:4378-4382.

Sun, S.-K. 1975. The disease cycle of rice bakanae disease in Taiwan. Proc. Natl. Sci. Counc. 8:245-256. (In Chinese with English abstract)

Sunder, and Satyavir. 1998. Vegetative compatibility, biosynthesis of GA3 and virulence of Fusarium moniliforme isolates from bakanae disease of rice. Plant Pathol. 47:767-772.

Tamura, S. 2012. Historical aspects of gibberellins. Pages 1-8 in: Gibberellins. N. Takahashi, B. O. Phinney, and J. MacMillan, eds. Springer-Verlag, New York.

Untergasser, A., Cutcutache, I., Koressaar, T., Ye, J., Faircloth, B. C., Remm, M., and Rozen, S. G. 2012. Primer3-New capabilities and interfaces. Nucleic Acids Res. 40:e115.

Venturini, G., Assante, G., Toffolatti, S. L., and Vercesi, A. 2011. Mating behavior of a Northern Italian population of Fusarium verticillioides associated with maize. J. Appl. Genet. 52:367-370.

Voigt, K., Schleier, S., and Brückner, B. 1995. Genetic variability in Gibberella fujikuroi and some related species of the genus Fusarium based on random amplification of polymorphic DNA (RAPD). Curr. Genet. 27:528535 .

Watanabe, T., and Umehara, Y. 1977. The perfect state of the causal fungus of bakanae disease of rice plants re-collected at Toyama. Trans. Mycol. Soc. Jpn. 18:136-142.

Yang, C.-D., Guo, L.-B., Li, X.-M., Ji, Z.-J., Ma, L.-Y., and Qian, Q. 2006. Analysis of QTLs for resistance to rice bakanae disease. Chin. J. Rice Sci. 20:657-659. (In Chinese)

Yu, K.-S., and Sun, S.-K. 1976. Ascospore liberation of Gibberella fujikuroi and its contamination of rice grains. Plant Prot. Bull. 18:319-332. (In Chinese with English abstract)

Yu, K.-S., and Sun, S.-K. 1977. Studies on inoculum potential and incubation period of rice bakanae disease. Plant Prot. Bull. 19:245-250. (In Chinese with English abstract) 